\title{
ON CERTAIN MINIMUM PROBLEMS IN THE THEORY OF CONVEX CURVES $\left({ }^{1}\right)$
}

\author{
BY \\ MARLOW SHOLANDER
}

Introduction. In Chapter I we describe a set of eight related problems. Solutions of five of these problems are known. Solutions for two of the remaining three problems are given in Chapters VI and VIII, and a partial solution of the third is given in Chapter IX. The general method of attack is developed in Chapters III and IV. This method centers around a process whereby a plane convex body is asymmetrized into one of a set of associated bodies called triarcs.

If difficulties occur in reading Chapter I, it is suggested that Chapter II be read first. Background material not found in the latter chapter is to be found in the well known book Theorie der konvexen Körper by Bonnesen and Fenchel [1]. References to this and other sources are made by numbers, corresponding to the list at the end of the paper, placed in brackets. The notation, say, Theorem III 1.1 refers to Theorem 1.1 in Chapter III of this paper. Attention is directed to new inequalities in Theorem VI 1.2 and in Theorem VIII 1.2.

\section{Chapter I. A SET of extremal problems}

In the set of quantities associated with a convex body we are principally concerned with its area $A$, perimeter $C$, diameter $D$, and thickness $E$. Inequalities which hold for pairs of these quantities are given below. The inequalities are sharp, in the sense that bodies exist for which equality holds. The phrase in parentheses following an inequality describes the set of bodies for which we have equality.

(1) $E \leqq D \quad$ (orbiforms).

(2) $\pi E \leqq C \quad$ (orbiforms).

(3) $2 D \leqq C \quad$ (segments).

(4) $C \leqq \pi D$ (orbiforms).

(5) $E^{2} \leqq 3^{1 / 2} A$ (equilateral triangles).

(6) $4 A \leqq \pi D^{2}$ (circles).

(7) $4 \pi A \leqq C^{2}$ (circles).

Inequalities involving more than two of the quantities are naturally more difficult to obtain, and problems involving lower bounds for areas prove particularly troublesome. In 1923, Kubota [6] found the following inequali-

Received by the editors December 1, 1951.

(1) Parts of this paper were written while the author was under contract to the Office of Naval Research. 
ties, all sharp. (Formulas (10), (11), and (12) are given incorrectly in Bonnesen and Fenchel $[1$, p. 81$]$.)

(8) $2\left(D^{2}-E^{2}\right)^{1 / 2}+2 E$ arcsin $E / D \leqq C$ (the convex hull of a circle and two symmetrically placed points).

(9) $C \leqq 2\left(D^{2}-E^{2}\right)^{1 / 2}+2 D$ arcsin $E / D$ (a body formed by removing from a circular body points outside two symmetrically placed secants).

(10) $2 A \leqq E\left(D^{2}-E^{2}\right)^{1 / 2}+D^{2}$ arcsin $E / D$ (the body of the previous inequality).

(11) $4 A \leqq 2 E C-\pi E^{2}$ (the convex hull of two circles of equal radius).

(12) $8 \phi A \leqq C(C-2 D \cos \phi)$, where $2 \phi D=C \sin \phi \geqq 0$ (the intersection of two circular bodies of equal radius).

Fukasawa [3] has described bodies which have maximum area for given $C, D$, and $E$. Favard [2], Hayashi [4], Kawai [5], Kubota [6, 7], and Yamanouti $[10]$ have found partial results for the principal remaining problems:

(13) The $(D, E)$ problem. To find the convex bodies of minimum $A$ for given $D$ and $E$.

(14) The $(C, E)$ problem. To find the convex bodies of minimum $A$ for given $C$ and $E$.

(15) The $(C, D)$ problem. To find the convex bodies of minimum $A$ for given $C$ and $D$.

By Blaschke's Selection Theorem, we know solutions of these problems exist. Thus, we may prove a body is the solution of one of these problems by eliminating all other bodies as possible solutions. This method is followed in this paper in solving problems (13) and (14).

Problem (15) seems appreciably more difficult than the other two. The partial results given in Chapter IX make reasonable the more detailed description of the solution given there as a conjecture.

\section{Chapter II. Definitions, notation, and known Results}

A point set is called convex if, for every pair of points in the set, the segment joining the points is also in the set. A convex body is a bounded closed convex point set. We are concerned below only with two-dimensional sets.

The boundary of a convex body $\Omega$, denoted by $\Omega^{0}$, is the set of points found in $\Omega$, but not in its interior. The perimeter, $C$, of $\Omega$ may be defined as the length of $\Re^{0}$.

The symbols $\cup$ and $\cap$ are used to represent, respectively, the operations of point set addition and multiplication. The convex hull of a closed set of points is defined as the product, or intersection, of all closed half-planes containing the set. The convex hull may also be defined as the smallest convex body containing the set. The intersection of a set of convex bodies is a convex body.

It is convenient to treat points in the plane as vectors. For sets $\mathfrak{F}$ and 
$(\mathcal{G}, \mathfrak{F}+\mathscr{B}$ is defined as the set of all points of the form $P+Q$ where $P$ is in $\mathfrak{F}$ and $Q$ is in $\$$. For a real number $\lambda, \lambda F$ is defined as the set of all points $\lambda P$ for $P$ in $\mathfrak{F}$. If $\mathfrak{F}$ and $\mathbb{S}$ are convex bodies, $\lambda \mathfrak{F}+\mu ß$ is a convex body. For a point $P$ we note particularly the interpretations of $\Omega+P,-\Omega$, and $2 P-\Omega$ as, respectively, a translation of $\Omega$, the reflection of $\Omega$ with respect to the origin, and the reflection of $\Omega$ with respect to $P$. In some of our work it will be apparent that we choose to make no distinction between $\Omega$ and one of its translations.

We call a line $t$ a supporting line of $\Omega$ if $t$ contains at least one point of $\Omega$ and if $\Omega$ is contained in one of the closed half-planes determined by $t$. If $P$, a point in $\Omega^{0}$, lies on the supporting line $t$ of $\Omega$, a line perpendicular to $t$ at $P$ is called a normal of $\Omega$. This normal is divided by $P$ into two rays, an inner and outer normal. If $\alpha$ is chosen as the angle of inclination of the outer normal, we assign to $t$ the direction $\alpha+\pi / 2$. Thus, the lines of support may be directed and their directions determine in an obvious fashion a positive ordering for points in $\AA^{0}$.

A supporting line $t$ containing more than one point of $\Omega^{0}$ contains a segment of points of $\Omega^{0}$. This segment is called an edge of $\Omega$. Points in $\Omega^{0}$ interior to no edge of $\Re$ are called extreme points of $\Re$. If a point $P$ of $\Omega^{0}$ is contained in more than one supporting line of $\Omega$, it is called a corner of $\Omega$. To each corner $P$ corresponds an infinite set of lines of support whose directions cover closed disjoint intervals of the form $(\alpha+2 n \pi, \beta+2 n \pi), n=0$, $\pm 1, \pm 2, \cdots$. Each of these intervals is called a maximal interval of directions of support at $P$. Lines of support corresponding to no interior value of a maximal interval of directions are called extreme supporting lines of $\Re$.

Given a convex body $\Omega$, to each direction $\alpha$ corresponds a supporting line $t_{\alpha}$. The pair of lines $t_{\alpha}$ and $t_{\alpha+\pi}$ are said to form a double line of support and this is denoted by $t_{\alpha} t_{\alpha+\pi}$. The distance between $t_{\alpha}$ and $t_{\alpha+\pi}$ is called the width of $\Omega$ in the direction $\alpha$ and is denoted by $B$ or, more precisely, by $B_{\alpha}$. The diameter $D$ and the thickness $E$ are defined, respectively, as the maximum and minimum of $B_{\alpha}$. A chord of $\Omega$ with an end point on each line of a double line of support $t_{\alpha} t_{\alpha+\pi}$ is called a major chord of $\Re$ and it may be directed by choosing as its angle of inclination a value between $\alpha-\pi$ and $\alpha$. If this direction is $\alpha-\pi / 2$, the major chord is called a double normal of $\Omega$. Major chords of length $D$ and $E$ are double normals. In a set of parallel chords of $\Omega$, a chord of maximum length is a major chord and it need, of course, be uniquely determined only in the sense of a vector. The end points of a major chord are called opposite points.

The statement that $\Im$ is supported by $\Omega$ or $\Omega$ supports $\Im$ implies that $\Im$ is contained in $\Re$ and that, $\Omega$ being fixed in position, no body of the form $\lambda \Im+P$ is contained in $\Re$ for $\lambda>1$. If $\Re$ contains $\Im$, a necessary and sufficient condition that $\Omega$ supports $\Im$ is that there exists no closed interval of directions of length $\pi$ free from the direction of a common supporting line of $\Im$ and $\Omega$. 
The convex hull of $\Im$ and a point $P$ is called a simple capping body of $\Im$. In general, $\Re$ is a capping body of $\Im$ if $\Omega$ contains $\Im$ and if all supporting lines of $\Omega$, with the exception of nonextreme lines of support on a set of corners of $\Omega$, are supporting lines of $\Im$. A capping body of $\Im$ may also be defined as the convex hull of $\Im$ and a set of points no two of which may be separated from $\Im$ by the same supporting line of $\Im$. Thus a capping body of $\Im$ consists of the union of $\Im$ and a set of disjoint caps. Each of these caps has as boundary a pair of segments and an arc from $\Im^{0}$. The segments, sides of the cap, intersect in a point called the peak of the cap. The arc from $\Im^{0}$ is called the base of the cap and its end points the end points of the cap. If the base of the cap is a circular arc, the cap is called a circle cap. A cap with peak $Q$ and end points $P$ and $R$ will be called cap $P Q R$ or cap $R Q P$.

The intersection of $\Omega$ with a closed half-plane is called a simple cutting body of $\Omega$. In general, a cutting body of $\Omega$ is defined as a convex body $\Im$ such that the set of points in $\Im^{0}$ but not in $\Im^{0} \cap \Omega^{0}$ has as closure the union of a set of chords of $\Omega$.

For the definition of a convergent sequence of convex bodies see Bonnesen and Fenchel [1]. Each such sequence has a uniquely determined convex body as limit. Blaschke's Selection Theorem states that in each uniformly bounded infinite set of convex bodies there exists a subset which may be ordered to form a convergent sequence. A function $f(\Omega)$ of a body $\Omega$ is, by definition, continuous if $\lim _{i \rightarrow \infty} \Omega_{i}=\Omega$ implies $\lim _{i \rightarrow \infty} f\left(\Omega_{i}\right)=f(\Omega)$. The quantities $A, C, D$, and $E$ are continuous functions of $\Omega$.

We say $\Omega$ has center $P$ if $2 P-\Omega=\Omega$. If $\Omega$ has a center $P$, to each direction there corresponds a major chord bisected by $P$. For each convex body $\Re$, the body $\Re-\Omega$ has the origin as center. The latter is called the vector body of $\Omega$ since its boundary consists of the terminal points of vectors which correspond to the major chords of $\Omega$.

If the thickness and diameter $D$ of $\Omega$ are equal, we called $\Omega$ an orbiform of width $D$ and, in this case, $\Omega^{0}$ is often called a curve of constant width. An orbiform has no edges and each of its major chords is a double normal. A body of diameter $D$ can always be imbedded in an orbiform of width $D$. The simplest orbiform not a circle is a Reuleaux triangle. This is constructed by taking an equilateral triangle $R S T$ of side $D$ and forming the intersection of the circles $C(R, D), C(S, D)$, and $C(T, D)$. Reuleaux polygons both regular and not regular with an odd number of sides, 5 or more, may be constructed in a somewhat similar fashion.

The centralization of $\Omega$ is defined as $(\Omega-\Omega) / 2$ and denoted by $\Omega^{*}$. The bodies $\Omega$ and $\Omega^{*}$ have, in each direction $\alpha$, the same width $B_{\alpha}$ and hence equal diameter and thickness. Moreover, $\Omega$ and $\Omega^{*}$ have equal perimeters. The area of $\Omega$ is no greater than the area of $\Omega^{*}$ and equality holds if and only if $\Omega$ has a center.

Nonsingular affine transformations preserve not only convexity but also 
double lines of support, major chords, and many other properties and relations. A proof is often simplified by the use of a judiciously chosen affine transformation.

\section{Chapter III. Center equivalence and heXagons}

We call two convex bodies center equivalent if they have the same centralization. Our primary problem, that of describing bodies of minimum area in sets of center equivalent bodies, is solved in the next chapter. In this chapter we take the preliminary steps. In $\S \S 1$ and IV 3 we follow a method of attack used by Lebesgue to solve a more specialized problem (see §IV 4).

1. The Lebesgue process. The process we wish to modify is found in Bonnesen and Fenchel $[1, \S 66]$ as well as in Lebesgue's papers $[8,9]$.

Consider a convex body $\Omega$ of area $A$. Let $\alpha_{1}, \alpha_{2}, \cdots$ be a fixed countable set of numbers contained in $(0, \pi)$ and everywhere dense in that interval. Let $t_{i} t_{i}^{\prime}$ be the double line of support corresponding to direction $\alpha_{i}$ and let $B_{i}$ be the width of $\Omega$ in this direction. The set of number pairs $\left(\alpha_{i}, B_{i}\right)$ determines $\Omega^{*}$ and hence determines the set of bodies center equivalent to $\Omega$. To each subscript $k \geqq 2$ corresponds a polygon $\mathfrak{B}_{k}$ circumscribed to $\Re$ and determined by $t_{i} t_{i}^{\prime}$ for $i=1,2, \cdots, k$. The sequence $\left\{\Re_{k}\right\}$ converges to $\Omega$. $\mathfrak{B}_{k+1}$ is a cutting body of $\mathfrak{B}_{k}$ formed by the removal of two similar triangles from $\mathfrak{B}_{k}$. Let $A_{k}$ be the area of $\mathfrak{B}_{k}$ and let $\Delta_{k}=A_{k}-A_{k+1}$. The lengths of the two edges of $\mathfrak{P}_{k+1}$ not contained in $\mathfrak{P}_{k}^{0}$ can easily be shown to have a sum dependent only on $\Omega^{*}$. If one of these lengths is zero, one of the similar triangles removed has area 0 and the other has an area $a_{k}$. In this case $\Delta_{k}=a_{k}$. If these lengths are equal, $\Delta_{k}=a_{k} / 2$. In all other cases $\Delta_{k}=\mu_{k} a_{k}$ where $1 / 2<\mu_{k}$ $<1$. We thus obtain the following theorem.

THEOREM 1.1. $A=A_{2}-\sum_{k=2}^{\infty} \mu_{k} a_{k}$ where, for $k=2,3, \cdots, 1 / 2 \leqq \mu_{k} \leqq 1$. The first equality holds if and only if $t_{k+1}$ and $t_{k+1}^{\prime}$ cut off congruent triangles from $\mathfrak{B}_{k}$. The second equality holds if and only if either $t_{k+1}$ or $t_{k+1}^{\prime}$ is a supporting line of $\mathfrak{P}_{k}$.

Upon closer examination of the proof we see that it is easy to establish other results.

THEOREM 1.2. The sum of the lengths of parallel edges of $\Omega$ depends only on $\Omega^{*}$. The perimeter of $\Omega$ equals the perimeter of $\Omega^{*}$. The sum of the radii of curvature of $\Omega^{0}$ at the end points of a major chord of $\Omega$ in a given direction $\alpha$ depends only on $\Re^{*}$. Furthermore, using an obvious notation, $\mathfrak{P}_{2}$ is congruent to $\left(\mathfrak{P}^{*}\right)_{2}$ and, for $k>2, \mathfrak{P}_{k}$ is center equivalent to $\left(\mathfrak{B}^{*}\right)_{k}$.

2. Hexagons. We use the following abbreviations. An $R$-hexagon is a regular hexagon. An $A$-hexagon is one which becomes an $R$-hexagon under a suitably chosen nonsingular affine transformation. A $C$-hexagon is one which has a center. A $P$-hexagon is one which has each pair of opposite sides 
parallel. A hexagon described by one of these four letters is assumed to be nondegenerate. Finally when one of these letters is preceded by the letter $C$ or $I$, we refer to a circumscribed or inscribed hexagon-e.g., an $R$-hexagon is an $I R$-hexagon of its circumscribed circle. It is known that every orbiform admits a $C R$-hexagon.

It is clear that $R$-hexagons are $A$-hexagons, $A$-hexagons are $C$-hexagons, and $C$-hexagons are $P$-hexagons. If a $P$-hexagon has one pair of opposite sides equal in length, it is a $C$-hexagon. If a $C$-hexagon has a major diagonal parallel to and twice the length of either disjoint side, it is an $A$-hexagon. An $A$-hexagon with equal sides or equal vertex angles is an $R$-hexagon.

A triangle and its reflection with respect to a point have either a parallelogram or $C$-hexagon as convex hull and either the null set, a parallelogram, or a $C$-hexagon as intersection. These $C$-hexagons are $A$-hexagons if and only if the point of reflection is the centroid of the triangle. The hexagon whose vertices are the midpoints of the sides of a $C$-hexagon is an $A$-hexagon.

From the discussion of $\S 1$ we see that hexagons center equivalent to a $C$-hexagon are $P$-hexagons and two $P$-hexagons are center equivalent if and only if their sides are respectively parallel and the corresponding sums of lengths of parallel sides are equal. A triangle is center equivalent to an $A$-hexagon.

A $P$-hexagon is formed by translating one of the double lines of support determined by opposite sides of its center equivalent $C$-hexagon. This translation increases the length of one of two parallel sides as it decreases the length of the other. (It can be shown that the absolute value of the difference of these lengths is proportional to the sine of a disjoint vertex angle - a result generalizing the sine law for triangles.) Indeed if we call a side of a $P$-hexagon long (short) when it is longer (shorter) than the opposite side, we have the following result.

THEOREM 2.1. The sides of a $P$-hexagon not a $C$-hexagon are alternately long and short.

Let $K L M N O P$ be a $C$-hexagon of area $A_{c}$. Let $K^{\prime} L^{\prime} M N^{\prime} O^{\prime} P$ be a center equivalent $P$-hexagon of area $A_{p}$. Let $K^{\prime} M O^{\prime}$ have area $A_{t}$. Let $A_{0}$ be the area of the triangle formed by passing lines through $K^{\prime}, M$, and $O^{\prime}$ parallel respectively to the disjoint sides of $K^{\prime} L^{\prime} M N^{\prime} O^{\prime} P$. (In the notation of $\S 1, A_{0}$ equals $\left(2 \mu_{2}-1\right) a_{2}$.) The following theorem is easily proved.

Theorem 2.2. $A_{0}=2 A_{t}-A_{p}=2\left(A_{c}-A_{p}\right)$.

CoRollary 2.3. In the set of all hexagons center equivalent to a P-hexagon, the hexagon of greatest area is the C-hexagon.

The following theorem is of some intrinsic interest. It supplements the known result that a hexagon (more generally, $n$-gon) of minimum area circum- 
scribed to a convex curve has the property that midpoints of its sides are points of contact with the curve.

THEOREM 2.4. Let $\AA^{*}$, not a segment, be a convex body with a center and let $m$ be the infimum of the areas of its circumscribed hexagons. There exists a $C C$ hexagon of $\Omega^{*}$ which has area $m$.

Proof. Since a $C P$-hexagon of $\Omega^{*}$ is a $C$-hexagon, it is sufficient to show that a $C P$-hexagon of $\Omega^{*}$ has area $m$. By Blaschke's Selection Theorem, a circumscribed hexagon $\mathfrak{S}$ of area $m$ exists. Since $\mathfrak{S}$ may be expressed as the limit of a sequence of circumscribed hexagons each of which has no two of its sides parallel, our task clearly reduces to the following: to show that if $\mathfrak{E}$ has no two of its sides parallel, there exists a $C P$-hexagon of $\Omega^{*}$ whose area is less than the area of $\mathfrak{S}$.

Let the origin $O$ be the center of $\Omega^{*}$. The intersection of $\mathfrak{S}$ and $-\mathfrak{S}$ is a circumscribed dodecagon $\&$ of $\Omega^{*}$ whose opposite sides are parallel. Let the sides of $\mathfrak{R}$ be denoted by $s_{i}, i=1,2, \cdots, 12$. Let $\mathfrak{S}(1,3,5,7,9,11)$, for example, be the hexagon formed by extending sides $s_{1}, s_{3}, s_{5}, s_{7}, s_{9}$, and $s_{11}$.

If $s_{i}$ is a side of $\mathfrak{S}, s_{i+6}$ is not a side of $\mathfrak{T}, i=1,2, \cdots, 6$, and conversely. We may further assume that no three consecutive sides of $\mathfrak{R}$ are sides of $\mathfrak{S}$. To see this, suppose $s_{1}, s_{2}$, and $s_{3}$ are sides of $\mathfrak{S}_{\text {. }}$. Then $s_{7}, s_{8}$, and $s_{9}$ are not, and side $s_{2}$ of $\mathfrak{S}$ may be replaced by side $s_{8}$ to obtain a hexagon of smaller area. On the other hand, at least two sides of $\mathfrak{S}$ are consecutive sides of $\mathfrak{l}$, for otherwise opposite sides of $\mathfrak{S}$ would be parallel. Let $s_{1}$ and $s_{2}$ be two such sides. It follows that $\mathfrak{S}$ is either $\mathfrak{S}(1,2,5,6,9,10)$ or $\mathfrak{S}(1,2,4,6,9,11)$. In either case by inspecting the areas found in the hexagons but not found in $\mathbb{R}$, it is seen that the area of $\mathfrak{S}$ plus the area of $-\mathfrak{S}$ exceeds the sum of the areas of the $C P$-hexagons $\mathfrak{S}(1,3,5,7,9,11)$ and $\mathfrak{S}(2,4,6,8,10,12)$. Hence at least one of these latter hexagons has smaller area.

With the aid of the following elementary lemma in establishing the relative sizes of various triangular areas, Theorem 2.6 may be proved very much as we proved Theorem 2.4.

LEMmA 2.5. If points $V$ and $W$ lie respectively on sides $P R$ and $S P$ of triangle $R S P$ and if $V S$ and $R W$ meet at $Q$, area $R S Q \geqq$ area $W V Q$. If $T$ lies inside triangle VWP, area $R S T>$ area $V W T$.

THEOREM 2.6. Let $\Omega^{*}$, not a segment, have a center and let $M$ be the supremum of the areas of its inscribed hexagons. There exists an IC-hexagon of $\Omega^{*}$ with area $M$.

We close the section by stating another theorem whose proof presents no difficulties.

TheOREM 2.7. If $P$ is the center of $\Omega^{*}$ and $\mathfrak{S}$ is an IC-hexagon of $\Omega^{*}$, then $P$ is the center of $\mathfrak{S}$. 
3. Flatness. We call a convex body $\Omega$ flat [strictly flat] (in direction $\alpha$ ) if for some $\alpha$ the sum of the lengths of edges of $\Omega$ in directions $\alpha$ and $\alpha+\pi$ is not less [is greater] than the length of the major chord of $\Omega$ in direction $\alpha$. Thus segments and trapezoids are strictly flat, points and triangles are flat, and circles and regular pentagons are not flat.

It is evident that flatness is preserved under affine transformations. By Theorem 1.2, it is also preserved under centralization: $\Omega^{*}$ is flat if and only if it has an edge whose length is not less than half the length of the parallel major chord.

THEOREM 3.1. If $\Omega^{*}$ is flat in three distinct directions, it is a segment or a $C$-hexagon strictly flat in at most one direction. Hence if $\Omega$ is flat in three distinct directions, it is a segment, a triangle, a trapezoid, or one of a class of $P$-hexagons. If $\Omega$ is flat in more than three directions, it is a segment.

Proof. Consider $\Omega^{*}$, not a segment, flat in three directions. We may subject $\Omega^{*}$ to a shear in a direction of flatness and compressions parallel and perpendicular to this direction so that $\Omega^{*}$ is carried into a body $\Im$ with the following properties: $\Im$ has a center $O ; \Im$ has an $I C$-hexagon $L M N P Q R$ with $R N$ the major chord of $\Im$ in direction $\alpha$ and with $L M$ and $Q P$ edges of $\Im$ parallel to $R N$; the perpendicular bisector of $R N$ bisects $L M$; the distance from $L M$ to $Q P$ is $2\left(3^{1 / 2}\right)$ and the length of $R N$ is 4 . By Theorem 2.7, $O$ is the center of $L M N P Q R$. It is sufficient to show $\Im$ is a $C$-hexagon strictly flat in at most one direction.

Let $\mathfrak{S}$, the $I R$-hexagon of $\Im$ which has $R N$ as major diagonal, have vertices $L_{0} M_{0} N P_{0} Q_{0} R$. Let $L_{1} M_{1} P_{1} Q_{1}$ be a parallelogram supporting $\Im$ such that $M_{1} P_{1}$ meets $\Im$ at $N$ and the edge $Q Q_{0} P_{0} P$ of $\Im$ lies on $Q_{1} P_{1}$. Since this parallelogram is flat in only two directions, in one of the directions of flatness of $\Im$ a supporting line of $\Im$ separates, say, vertex $P_{1}$ from $\Im$. Let this line of support meet $P P_{1}$ at $P_{2}$ and $N P_{1}$ at $N_{2}$. Let $M_{0} N$ extended meet $P_{2} N_{2}$ extended at $N_{1}$. Select point $S$ so that $P_{0} S$ is parallel to $P_{2} N_{2}$ and so that $S$ lies either on $O N$ or $N N_{1}$. Twice the length of $P_{0} S$ is the length of the major chord of $\mathfrak{S}$ in the direction $P_{2} N_{2}$. Let $m$ be the length of the major chord of $\Im$ in direction $P_{2} N_{2}$ and let $n$ be the length of the edge of $\Im$ lying in $P_{2} N_{2}$.

By the definition of flatness, $m \leqq 2 n$. But it is evident that $n \leqq P_{2} N_{2}$ $\leqq P_{2} N_{1} \leqq P_{0} S \leqq m / 2$ and hence the inequalities may be replaced by equal signs. There are now two cases. If $S$ is on $O N, \Im$ is the hexagon $L M N P Q R$ and $\Im$ is strictly flat only in direction $\alpha$. If $S$ is not, $\Im$ is the hexagon $L_{0} M_{0} S P_{0} Q_{0} T$, where $T=-S$, and $\Im$ is strictly flat only in direction $M_{0} S$.

The following theorem is easily proved.

Theorem 3.2. Let $\Omega^{*}$ have center $O$ and let $P$ be a point on $\Omega^{* 0}$. Then $\Omega^{* 0} \cap\left(\Omega^{* 0}+P\right)$ consists of two points unless $\Omega^{*}$ is strictly flat in direction OP, in which case it consists of two segments parallel to OP. The width of $\Omega^{*}$ 
$\cap\left(\Omega^{*}+P\right)$ in direction $O P$ equals the width of $\Omega^{*}$ in direction $O P$ if and only if $\Omega^{*}$ is flat in that direction.

4. $I A$-hexagons of $\Omega^{*}$. In this section $\Omega^{*}$ denotes a convex body, not a segment, which has the origin $O$ as center.

TheOREM 4.1. If $L$ lies on $\Omega^{* 0}, L$ is a vertex of an IA-hexagon of $\Omega^{*}$. This hexagon is unique when $\Omega^{*}$ is not flat in direction $O L$. A side of the hexagon lies on an edge of $\Omega^{*}$ if and only if $\Omega^{*}$ is flat in the direction of that side.

Proof. Let $R=-L$. Let $M$ be a point of the component of $\Omega^{* 0} \cap\left(\Omega^{* 0}+L\right)$ on the positively directed arc $L R$ of $\Omega^{* 0}$. Let $N=M-L, S=-M$, and $T$ $=-N$. Then $T$ belongs to the other component of $\Omega^{* 0} \cap\left(\Omega^{* 0}+L\right)$ and both $N$ and $S$ belong to $\Re^{* 0} \cap\left(\Omega^{* 0}-L\right)=-\left[\Omega^{* 0} \cap\left(\Omega^{* 0}+L\right)\right]$.

It is evident that LMNRST is an $I C$-hexagon of $\Omega^{*}$ with $O$ as its center. Since $N M$ is parallel to $R L$ and half as long, $L M N R S T$ is an $I A$-hexagon of $\Omega^{*}$. Assume $L M^{\prime} N^{\prime} R^{\prime} S^{\prime} T^{\prime}$ is another $I A$-hexagon of $\Omega^{*}$. By Theorem 2.7, this also has $O$ as center. Clearly $R=R^{\prime}$ and $2 M N=L R=L^{\prime} R^{\prime}=2 M^{\prime} N^{\prime}$. To avoid having one of these points interior to $\Omega^{*}$, we must assume $M, N, M^{\prime}$, and $N^{\prime}$ are collinear. Either the hexagons are identical or it follows that $M N$ is on an edge of $\Re^{*}$ and $\Omega^{*}$ is flat in direction $M N$.

Corollary 4.2. If $L$ lies on $\Omega^{* 0}, L$ is a vertex of an inscribed triangle $L N S$ of $\Omega^{*}$ which has $O$ as centroid. This triangle is unique if $\Omega^{*}$ is not flat in direction $O L$.

Corollary 4.3. If $\Omega$ is not flat, to each direction $\theta$ corresponds a unique pair of directions $\phi$ and $\psi$ such that the sum of the vectors determined by major chords in these directions is zero.

The corollaries above have obvious analogues in corollaries for the next theorem. The remainder of the section is devoted to the proof of the theorem.

Theorem 4.4. The IA-hexagons of $\Omega^{*}$ may be placed in one-many correspondence with real numbers $\beta,-\infty<\beta<\infty$, so that:

(i) as $\beta$ increases, the hexagons turn continuously in the positive directioni.e., the inclinations of the rays from $O$ to the vertices of the hexagons are continuous nondecreasing functions of $\beta$.

(ii) for each real $\beta_{0}$, the hexagons are in one-one correspondence with the values $\beta_{0} \leqq \beta<\beta_{0}+\pi / 3$.

Consider an $I A$-hexagon of $\Re^{*}$, say, $V_{10} V_{30}^{\prime} V_{20} V_{10}^{\prime} V_{30} V_{20}^{\prime}$. Let the rays $O V_{10}, O V_{20}$, and $O V_{30}$ have respectively inclinations $\beta_{10}, \beta_{20}$, and $\beta_{30}$. We assume first that $\Omega^{*}$ is flat in direction $\beta_{10}$ and we may assume $V_{30}^{\prime}$ is an end point of an edge of $\Omega^{*}$. Let $V_{21}$ be the other end point. A point $V_{2}$ in the closed arc $V_{20} V_{21}$ is a continuous function of $\beta_{2}$ as $\beta_{2}$ increases from $\beta_{20}$ to, say, $\beta_{21}$. The points $V_{2}$ are in one-one correspondence with the $I A$-hexagons 
$V_{10} V_{3}^{\prime} V_{2} V_{10}^{\prime} V_{3} V_{2}^{\prime}$ which have $V_{10}$ as a vertex. Let $\beta_{3}$ be the inclination of $O V_{3}$ and let $3 \beta=\beta_{10}+\beta_{2}+\beta_{3}$. We note that $\beta_{2}$ and $\beta_{3}$ are strictly increasing functions of $\beta$.

If $\Omega^{*}$ is not flat in a direction $\beta_{i 0}$, we choose a point $V_{11}$ on $\AA^{* 0}$, a small positive distance from $V_{10}$, and let $\beta_{11}$ be the inclination of $O V_{11}$. By Theorem 3.1 , we may assume no direction $\beta_{1}$ in the closed interval from $\beta_{10}$ to $\beta_{11}$ is a direction of flatness of $\Omega^{*}$. The point $V_{11}$ determines a unique $I A$-hexagon $V_{11} V_{31}^{\prime} V_{21} V_{11}^{\prime} V_{31} V_{21}^{\prime}$. Since $V_{10}+V_{20}+V_{30}=0=V_{11}+V_{21}+V_{31}$, the vector displacements $V_{10} V_{11}, V_{20} V_{21}$, and $V_{30} V_{31}$ sum to zero. Let lines of support to $\Omega^{*}$ at $V_{10}, V_{20}$, and $V_{30}$ have respective directions $\theta, \phi$, and $\psi$, where $\theta<\psi$ $-\pi<\phi<\theta+\pi<\psi<\phi+\pi<\theta+2 \pi$. Since $V_{10} V_{11}$ has a direction in the interval $(\theta, \psi-\pi)$ and $V_{30} V_{31}$ has a direction in one of the intervals $(\theta, \psi-\pi),(\psi, \phi+\pi)$, we see that $V_{20} V_{21}$ has a direction in the interval $(\phi, \theta+\pi)$ and hence $V_{30} V_{31}$ has a direction in $(\psi, \phi+\pi)$. To summarize, as $V_{1}$ moves positively on $\Omega^{* 0}$, vertices $V_{2}$ and $V_{3}$ of an $I A$-hexagon $V_{1} V_{3}^{\prime} V_{2} V_{1}^{\prime} V_{3} V_{2}^{\prime}$ also move positively. As before, let $\beta_{i}$ be the inclination of $O V_{i}$ and $3 \beta=\beta_{1}+\beta_{2}+\beta_{3}$. Each $\beta_{i}$ in this case is a strictly increasing function of $\beta$.

We may clearly proceed thus around $\Omega^{* 0}$ (in either direction) assigning to each $I A$-hexagon of $\Omega^{*}$ a value $\beta$. When $\beta$ has increased by $\pi / 3$ we obtain the original hexagon with the vertices relettered. After $\beta$ has increased by $2 \pi$, we obtain the original hexagon with the original lettering.

5. Families of circumscribed hexagons. Consider again a convex body $\Omega$, not a segment, and its centralization $\Omega^{*}$. Our first task is to select a "continuously turning family of $C C$-hexagons" of $\Omega^{*}$ which have as points of contact the vertices of an $I A$-hexagon. Each $C C$-hexagon and an associated $I A$-hexagon may then be thought of as a member of a continuously turning family of "hexagonal configurations."

Let $\mathfrak{R}_{0}$ be an $I A$-hexagon of $\Omega^{*}$ with vertices $V_{10} V_{30}^{\prime} V_{20} V_{10}^{\prime} V_{30} V_{20}^{\prime}$ such that, in the notation of $\S 4, \beta_{i 0}$ is not a direction of flatness of $\AA^{*}$ and $V_{i 0}$ is not a corner of $\Omega^{*}, i=1,2,3$. Let $3 \beta_{0}=\beta_{10}+\beta_{20}+\beta_{30}$, let the supporting line of $\Omega^{*}$ at $V_{i 0}$ have direction $\gamma_{i 0}$, and let $3 \gamma_{0}=\gamma_{10}+\gamma_{20}+\gamma_{30}$. These directions are uniquely determined by requiring that $\beta_{10} \leqq \gamma_{20}-\pi, \gamma_{30} \leqq \beta_{10}+2 \pi$, and $\gamma_{10}$ $<\gamma_{20}<\gamma_{30}$.

To each $\beta$ in the interval $\beta_{0} \leqq \beta<\beta_{0}+\pi / 3$ corresponds a unique $I A$ hexagon, \&, with vertices $V_{1} V_{3}^{\prime} V_{2} V_{1}^{\prime} V_{3} V_{2}^{\prime}$. To each \& corresponds one or more polygons $\Im, C C$-hexagons or parallelograms, formed by the lines of support at vertices of $\mathbb{R}$. The set of all such polygons is in one-one correspondence with number triples $\left(\gamma_{1}, \gamma_{2}, \gamma_{3}\right)$ formed by choosing $\gamma_{i}$ as the direction of the supporting line resting on $V_{i}$ and requiring that $\gamma_{1}<\gamma_{2}<\gamma_{3}$ and $3 \gamma_{0} \leqq \gamma_{1}+\gamma_{2}+\gamma_{3}<3 \gamma_{0}+\pi$. Let $3 \gamma=\gamma_{1}+\gamma_{2}+\gamma_{3}$. To each value of $\beta$ in the interval $\left(\beta_{0}, \beta_{0}+\pi / 3\right)$ corresponds a value $\gamma$ or an interval of values $\gamma$. Let this correspondence determine points $(\beta, \gamma)$ in a coordinate plane and let $\&$ be the set of all such points. 
(S) has roughly the appearance of a graph of a monotone increasing function of $\beta$. That this is not strictly true is due to the possible presence in (B) of :

(i) Vertical segments. Such a segment occurs when, for a given $\beta$, one or more vertices of $\mathfrak{R}$ is a corner of $\Omega^{*}$.

(ii) Horizontal segments. One of these segments is determined by values of $\beta$ corresponding to a direction $\beta_{1}$ in which $\Omega^{*}$ is strictly flat. The polygons $\Im$ corresponding to interior points of these intervals are parallelograms.

(iii) Rectangles. These occur when we have situation (ii) and when, in addition, $V_{1}$ is a corner of $\Omega^{*}$.

Let $\left(S_{0}\right.$ be the subset of $(S)$ formed by removing from rectangles of $(S)$ points which lie neither on an upper nor on a left-hand edge. $\mathscr{S}_{0}$ is a simple arc and to each point of $\mathrm{S}_{0}$ not in the interior of a vertical segment corresponds a unique triple $\left(\gamma_{1}, \gamma_{2}, \gamma_{3}\right)$ or unique polygon $\Im$. We fail to have such a unique correspondence for points in vertical segments of $B_{0}$ when we have situation (i) and when more than one of the vertices $V_{1}, V_{2}$, and $V_{3}$ are corners of $\Omega^{*}$. We may obtain uniqueness, and also insure that we remove no parallelogram from the set of $\mathfrak{Y}$ under consideration, by discarding a properly selected subset of the triples. (This may be done by requiring that the lines of support at $V_{i}$ rotate in turn, in some prescribed order.)

Clearly $\left(\beta_{0}, \gamma_{0}\right)$ is the lowest point of $\mathscr{S}_{0}$ and $\left(\beta_{0}+\pi / 3, \gamma_{0}+\pi / 3\right)$ is its highest point. Let $2 \alpha_{0}=\beta_{0}+\gamma_{0}$ and, in general, let $2 \alpha=\beta+\gamma$. Both $\beta$ and $\gamma$ are monotone increasing functions of $\alpha$ in the interval $\left(\alpha_{0}, \alpha_{0}+\pi / 3\right)$. Translating $\mathfrak{S}_{0}$ parallel to the line $\beta=\gamma$ in the $(\beta, \gamma)$ plane by distances which are multiples of $\pi 2 /{ }^{1 / 2} 3$, we may extend the correspondences considered to other values of $\beta, \gamma$, and $\alpha$.

In summary we may state an extension of Theorem 4.4.

THEOREM 5.1. The set of real numbers $-\infty<\alpha<\infty$ may be placed in manyone correspondence with a set of hexagonal configurations $(\mathfrak{R}, \Im)$ where $\mathfrak{R}$ is an $I A$-hexagon of $\Omega^{*}$ and $\Im$ is an associated $C C$-hexagon, or circumscribed parallelogram, such that:

(i) as $\alpha$ increases, $(\mathcal{R}, \Im)$ turns continuously in such a way that lines in the configuration either turn positively or remain at rest.

(ii) for each real $\alpha_{0}$, there is a one-one correspondence between the values $\alpha_{0} \leqq \alpha<\alpha_{0}+\pi / 3$ and the set of configurations ( $\left.\mathbb{R}, \Im\right)$. The configuration corresponding to $\alpha=\alpha_{0}+\pi / 3$ is the configuration for $\alpha=\alpha_{0}$ with the vertices relettered.

The preceding theorem justifies our using the following notation. The hexagon $\&$ corresponding to $\alpha$ will be denoted in the remainder of the paper as $I A(\alpha)$ of $\Omega^{*}$. The hexagon or parallelogram $\Im$ is denoted as $C C(\alpha)$ of $\Omega^{*}$.

To each $C C(\alpha)$ of $\Omega^{*}$ corresponds a circumscribed polygon of $\Omega$ whose sides are parallel to the sides of $C C(\alpha)$. This is denoted as $C P(\alpha)$ of $\Omega$. If $C C(\alpha)$ of $\Omega^{*}$ is a parallelogram, $C P(\alpha)$ of $\Omega$ is a congruent parallelogram. Otherwise 
$C P(\alpha)$ is a $C P$-hexagon of $\Omega$. A $C P(\alpha)$ of $\Omega$ congruent to $C C(\alpha)$ of $\Omega^{*}$ is called a $C C$-support of $\Omega$.

TheOREM 5.2. Each convex body $\Re$ has at least one CC-support.

Proof. It is clear that if $\Omega$ is flat, at least one $C P(\alpha)$ of $\Omega$ is a parallelogram and the theorem holds. If $\Omega^{*}$ is not flat, it is sufficient to show some $C P(\alpha)$ of $\Omega$ is a $C C$-hexagon of $\Omega$ since, by Theorem 1.2, CP( $\alpha)$ is known to be center equivalent to $C C(\alpha)$ of $\Omega^{*}$. Let $C P(\alpha)$ of $\Omega$ have vertices $L M N R S T$. For $\alpha=\alpha_{0}$, we may assume that $L M$ is short and $M N$ is long (see Theorem 2.1). But for $\alpha=\alpha_{0}+\pi / 3$ (see Theorem 5.1), $L M$ is long and $M N$ is short. As $C P(\alpha)$ deforms continuously from the first to the second of these positions, for some intermediate $\alpha$ we have $L M=R S$ and, for that $\alpha, C P(\alpha)$ of $\Omega$ is a $C$-hexagon.

\section{Chapter IV. Triarcs}

In this chapter we define triarcs and show that to each convex body there corresponds an infinite set of center equivalent triarcs. We show further that, in the set of all bodies center equivalent to the given body, a body of minimum area is a triarc.

1. Properties of $n$-arcs. We call $\Omega$ a 1 -arc if $\Omega$ is a segment (or a point). We call $\Omega$ a $k$-arc, $k>1$, if $\Omega$ is not an $n$-arc, $n<k$, and if there exist points $P_{1}, P_{2}, \cdots, P_{k}$ on $\Re^{0}$ such that each double line of support of $\Re$ has one or more of these points as a point of contact. The points $P_{1}, P_{2}, \cdots, P_{k}$ are called vertices of $\Omega$ and, assuming they are positively ordered, we refer to $\Omega$ as the $k$-arc $P_{1} P_{2} \cdots P_{k}$. A triarc is defined as a $k$-arc, $k=1,2$, or 3 .

Thus triangles, quadrilaterals, and semicircles are 2 -arcs, and Reuleaux triangles and $P$-hexagons are 3 -arcs. A Reuleaux $n$-gon is an $n$-arc. A regular $n$-gon is an $[(n+1) / 2]$-arc. The property of being a $k$-arc is obviously preserved under nonsingular affine transformations.

We have temporary need of the following notation in discussing the $k$-arc $\Re, P_{1} P_{2} \cdots P_{k}$, where $k \geqq 2$. Let $t_{\alpha}$ be the supporting line of $\Re$ in direction $\alpha$. For $P$ on $\AA^{0}$, let $I(P)$ be a maximal interval of directions of support at $P$. This has the form $\alpha_{1} \leqq \alpha \leqq \alpha_{2}$, where $\alpha_{2}<\alpha_{1}+\pi$ and where for $\alpha_{2}<\alpha<\alpha_{1}+2 \pi$ line $t_{\alpha}$ does not pass through $P$. We have $\alpha_{1} \neq \alpha_{2}$ if and only if $P$ is a corner. Let $I_{n}(P)$, for $n=0, \pm 1, \pm 2, \cdots$, denote the closed interval $\left(\alpha_{1}+n \pi\right.$, $\left.\alpha_{2}+n \pi\right)$. Let $\Sigma_{i}^{\prime}=\bigcup_{n} I_{2 n+1}\left(P_{i}\right), \Sigma_{i}^{\prime \prime}=\bigcup_{n} I_{2 n}\left(P_{i}\right)$, and $\Sigma_{i}=\Sigma_{i}^{\prime} \cup \Sigma_{i}^{\prime \prime}$.

The following lemma follows trivially from these definitions.

Lemma 1.1. $\Sigma_{i}^{\prime} \cap \Sigma_{i}^{\prime \prime}=0$. For $i \neq j, P_{i} P_{j}$ is an edge of $\Omega$ if and only if $\Sigma_{i}^{\prime} \cap \Sigma_{j}^{\prime} \neq 0\left(\Sigma_{i}^{\prime \prime} \cap \Sigma_{j}^{\prime \prime} \neq 0\right)$. Vertices $P_{i}$ and $P_{j}$ are opposite points if and only if $\Sigma_{i}^{\prime} \cap \Sigma_{j}^{\prime \prime} \neq 0$. In general $\Sigma_{i}^{\prime} \cap \Sigma_{j}^{\prime \prime}$ is either null or a set of points of the form $\alpha_{0}+2 n \pi, n=0, \pm 1, \cdots$. 
LEMMA 1.2. The union of $\Sigma_{i}$ for any proper subset of indices $i=1,2, \cdots, k$ does not cover the interval $-\infty<\alpha<\infty$. The vertices $P_{i}, i=1,2, \cdots, k$, are distinct. Each vertex is a corner of $\Omega$.

Proof. The first two statements are immediate consequences of our insistence that a $k$-arc not be an $n$-arc, $n<k$. The last statement holds for the same reason. If $P_{1}$, say, is not a corner of $\Omega$ and if $t_{\alpha}$ rests on $P_{1}$, consider a sequence of directions $\left\{\alpha_{m}\right\}$ having $\alpha$ as limit. For some $j \neq 1$ and for some subsequence $\left\{\alpha_{m_{i}}\right\}$ of $\left\{\alpha_{m}\right\}$, the supporting line either in direction $\alpha_{m_{i}}$ or in direction $\alpha_{m_{i}}+\pi$ passes through $P_{j}$ for all $i$. It follows that $t_{\alpha+\pi}$ passes through $P_{j}$ and that $P_{1}$ may be removed from the set of vertices of $\Omega$.

We state another trivial lemma.

Lemma 1.3. $\bigcup_{i=1}^{k} \Sigma_{i}$ covers the interval $-\infty<\alpha<\infty$. The sum of the interior angles at corners $P_{1}, P_{2}, \cdots, P_{k}$ is at most $(k-1) \pi$.

Lemma 1.4. In the set of points opposite a vertex $P_{i}$ there exists either 0,1 , or 2 vertices. These possibilities correspond respectively to the cases where, of the pair of line segments joining $P_{i}$ to its adjacent vertices, we find both, one, or neither in the set of edges of $\Omega$. In the last case the segment joining the two vertices opposite $P_{i}$ is not an edge of $\Omega$.

Proof. We make use of the first statements in Lemmas 1.2 and 1.3. Consider, for example, the case where neither $P_{1} P_{2}$ nor $P_{2} P_{3}$ is an edge of $\Omega$. For $i=1,2,3$, let $I_{0}\left(P_{i}\right)$ be the interval $\left(\alpha_{i}, \beta_{i}\right)$. We have $\beta_{1}<\alpha_{2}<\beta_{2}<\alpha_{3}$. There exists an $\epsilon>0$ and an index $j$ such that $\left(\alpha_{2}-\epsilon, \alpha_{2}\right)$ is a subset of $\Sigma_{j}^{\prime}$, for otherwise in these directions double lines of support rest on no vertex. Thus $P_{2}$ is opposite $P_{j}$. Similarly, $P_{2}$ is opposite a vertex $P_{k}$ such that $\beta_{2} \in \Sigma_{k}^{\prime}$. No vertex $P_{n}$ is between $P_{j}$ and $P_{k}$ for this implies $\Sigma_{n} \subset \Sigma_{2}$. Moreover $h \neq j$, for otherwise $\Sigma_{2} \subset \Sigma_{j}$. Finally, if $P_{j} P_{k}$ is an edge of $\Omega$, we have the contradiction $\Sigma_{2} \subset \Sigma_{j} \cup \Sigma_{k}$.

To summarize the preceding lemmas in the shortest possible way we introduce a new definition. It is left to the reader to make obvious interpretations such as $P_{1}$ for $P_{k+1}$. A linkage of one vertex is defined as a vertex $P_{i}$ for which neither $P_{i-1} P_{i}$ nor $P_{i} P_{i+1}$ is an edge of $\Omega$. For $r>1$, a linkage of $r$ vertices is defined as a set of vertices $P_{i}, P_{i+1}, \cdots, P_{i+r-1}$ such that:

(i) $P_{i+j} P_{i+j+1}$ is an edge of $\Omega, j=0,1, \cdots, r-2$.

(ii) Neither $P_{i-1} P_{i}$ nor $P_{i+r-1} P_{i+r}$ is an edge of $\Omega$.

THEOREM 1.5. The set of vertices of a $k$-arc is partitioned uniquely into subsets forming linkages. These linkages may be placed in a unique cyclic order by starting with a linkage $L_{1}$, finding the linkage $L_{2}$ whose first vertex is opposite the last vertex of $L_{1}$, and so on. In a linkage of more than one vertex the terminal vertices are opposite exactly one vertex and the interior vertices are opposite no vertex. If $k>1$, the vertex in a linkage of one vertex is opposite exactly two vertices. 
THEOREM 1.6. The number of linkages formed by the vertices of $a k$-arc is always odd. If $k$ is even, there is at least one edge of the $k$-arc having vertices as end points.

Proof. The second statement follows from the first. Let $P_{1}$ be the first vertex in some linkage $L$ and let $\alpha_{1}$ be the smallest value in $I_{0}\left(P_{1}\right)$. Since directions sufficiently close to $\alpha_{1}$ and less than $\alpha_{1}$ belong to no set $\Sigma_{i}^{\prime \prime}$ (see Lemma 1.1), they belong to some set $\Sigma_{i}^{\prime}, i \neq 1$. If $P_{i}$ is a vertex in $L$, the vertices of $\Omega$ form a single linkage. If not, there are, say, $r$ linkages whose vertices correspond to directions of support in the closed interval $\left(\alpha_{1}, \alpha_{1}+\pi\right)$. The $r-1$ intervals of directions remaining in $\left(\alpha_{1}, \alpha_{1}+\pi\right)$ are associated with $r-1$ linkages whose vertices correspond to directions of support in the interval $\left(\alpha_{1}+\pi, \alpha_{1}+2 \pi\right)$. There are thus $2 r-1$ linkages.

TheOREm 1.7. A convex body $\Omega$ is a 2-arc if and only if $\Omega$ rests on two adjacent vertices of one of its circumscribed parallelograms.

Proof. The sufficiency is obvious. To prove necessity, consider a 2-arc $R S$. By Theorem 1.6, RS is an edge of $\Omega$ and $S$ (the last vertex in the linkage) is opposite $R$ (the first vertex in the "next" linkage). The desired parallelogram has sides parallel to $R S$ and to the direction of a double line of support resting on $R$ and $S$.

TheOREM 1.8. A convex body $\Omega$ is a 3-arc if and only if $\Omega$ has a CP-hexagon $\mathfrak{S}$ such that one of the following holds:

(i) $\Omega$ rests on 3 alternate vertices of $\mathfrak{W}$.

(ii) $\Re$ rests on 3 adjacent vertices of $\mathfrak{S}$.

Proof. Sufficiency of the condition is obvious. In proving necessity we first note, by Theorem 1.6, that vertices $R, S$, and $T$ of $\Omega$ form either 3 linkages of one vertex or one linkage of 3 vertices. In the first case, by Theorem 1.5, we have each vertex opposite the other two. The desired $C P$ hexagon has sides parallel to double lines of support resting simultaneously on pairs of vertices. In the second case, the reasoning is similar to that used in proving Theorem 1.7 .

The following corollaries are established by simple arguments. In connection with the first, we note that $\Omega$ may not be a triarc even though it rests on vertices $L, M, R$, and $S$ of a $C P$-hexagon $L M N R S T$.

COROLLARY 1.9. The set of vertices of a $k$-arc $\Omega, k=2$ or 3 , is unique if and only if $\Omega$ does not rest on $k+1$ vertices of a circumscribed polygon with the property of Theorem 1.7 or Theorem 1.8 .

COROLLARY 1.10. The only triarcs with centers are segments, parallelograms, and C-hexagons.

A triarc is called proper if it is a 2 -arc, or if it is a 3 -arc resting on alter- 
nate vertices of one of its $C P$-hexagons. If the hexagon of Theorem 1.8 is a $C P(\alpha)$ of $\Omega$, it is called a principal $C P(\alpha)$ of $\Omega$. If it is also a $C C$-support of $\Omega$, it is called a principal $C C$-support. If the parallelogram of Theorem 1.7 is a $C P(\alpha)$ of $\Omega$, it is both a principal $C P(\alpha)$ of $\Omega$ and a principal $C C$-support.

2. Triarcs and center equivalence. In this section the convex body $\Omega^{*}$ has the origin $O$ as center. We consider first an $I A(\alpha)$ of $\Omega^{*}$, say $V_{1} V_{3}^{\prime} V_{2} V_{1}^{\prime} V_{3} V_{2}^{\prime}$, such that $\Omega^{*}$ is not flat in the direction of a side of $I A(\alpha)$. Then $C C(\alpha)$ of $\Omega^{*}$ is a hexagon $W_{1} W_{3}^{\prime} W_{2} W_{1}^{\prime} W_{3} W_{2}^{\prime}$ where $W_{1} W_{3}^{\prime}$ rests on $V_{1}$ and so on.

Let $N=W_{1}^{\prime}+V_{1}$ and $P=W_{2}^{\prime}+V_{3}^{\prime}$. Let $(1 / 2) T(\alpha)$ be the convex body formed by the intersection of $\Omega^{*}, \Omega^{*}+V_{1}$, and $\Omega^{*}+V_{3}^{\prime}$ (see Fig. 1). Thus $V_{1}, V_{3}^{\prime}$, and $O$ are corners of $(1 / 2) T(\alpha)$ and the boundary of $(1 / 2) T(\alpha)$ is made up of arcs $V_{1} V_{3}^{\prime}, V_{3}^{\prime} O$, and $O V_{1}$ which are translations, respectively, of arcs $V_{1} V_{3}^{\prime}, V_{2} V_{1}^{\prime}$, and $V_{3} V_{2}^{\prime}$ of $\Omega^{* 0}$.

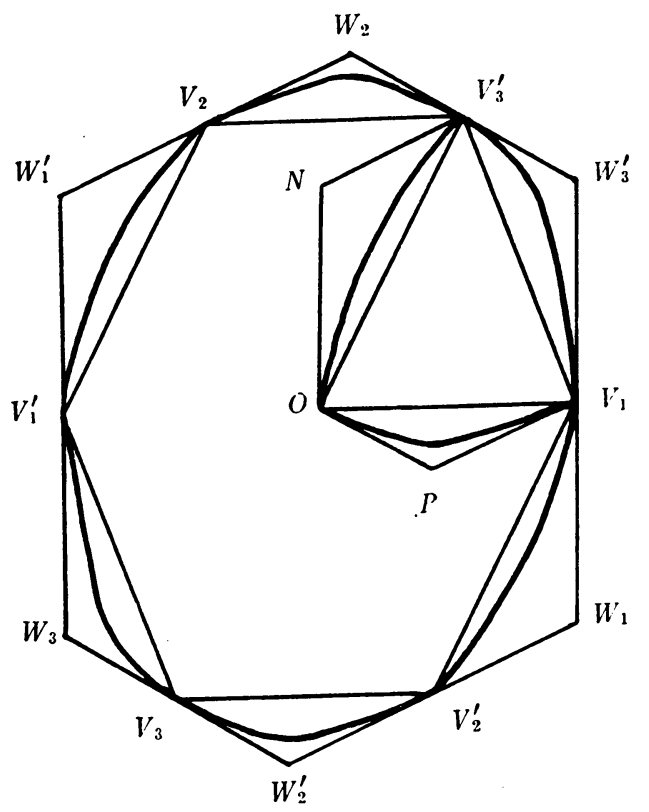

Fig. 1

THEOREM 2.1. If $\Omega^{*}$ is not flat in the direction of a side of $I A(\alpha)$ of $\Omega^{*}$, $T(\alpha)$ is a proper 3-arc center equivalent to $\Omega^{*}$. $C P(\alpha)$ of $T(\alpha)$, a principal $C P(\alpha)$ of $T(\alpha)$, is a principal CC-support if and only if vertices of $I A(\alpha)$ bisect sides of $C C(\alpha)$ of $\AA^{*}$.

Proof. It is clear that $T(\alpha)$ is a proper 3-arc. Consider a double line of support resting on points $V$ and $-V$ of $\Omega^{* 0}$ where $V$ lies on the arc, say, $V_{3}^{\prime} V_{2}$. A parallel double line of support to $(1 / 2) T(\alpha)$ meets its boundary 
at $V_{3}^{\prime}$ and at a point $Q$ on arc $O V_{1}$. By construction, $V_{3}^{\prime} Q$ is parallel to the segment from $V$ to $-V$ and has half its length. Thus $\Omega^{*}$ and $T(\alpha)$ are center equivalent. To complete the proof, we note that $O P V_{1} W_{3}^{\prime} V_{3}^{\prime} N$ is $C P(\alpha)$ of $(1 / 2) T(\alpha)$. This is a $C$-hexagon if and only if, say, $O N=V_{1} W_{3}^{\prime}$ or $W_{1} V_{1}=V_{1} W_{3}^{\prime}$. This shows, incidentally, that if a vertex of $I A(\alpha)$ of $\Omega^{*}$ bisects a side of $C C(\alpha)$ of $\Omega^{*}$, all vertices of $I A(\alpha)$ have this property.

In finding relations between areas of bodies considered in Fig. 1, we let triangle $O V_{1} V_{3}^{\prime}$ have area $t,(1 / 2) T(\alpha)$ have area $s+t, O P V_{1} W_{3}^{\prime} V_{3}^{\prime} N$ have area $r+s+t, \Omega^{*}$ have area $A^{*}, I A(\alpha)$ of $\Omega^{*}$ have area $A_{i}, C C(\alpha)$ of $\Omega^{*}$ have area $A_{c}, T(\alpha)$ have area $T$, and $C P(\alpha)$ of $T(\alpha)$ have area $T_{c}$. Then $t>0$, $r \geqq 0, s \geqq 0$, and we have $A_{i}=6 t, A^{*}=6 t+2 s, A_{c}=6 t+2 s+2 r, T=4 t+4 s$, and $T_{c}=4 t+4 s+4 r$. Thus in addition to $A_{i} \leqq A^{*} \leqq A_{c}$ and $T \leqq T_{c}$, we have $6 A_{c}=4 A_{i}+3 T_{c}, 6 A^{*}=4 A_{i}+3 T$, and (cf. Corollary 3.3) $2 A^{*} \leqq 3 T$.

These results are in part duplicated by Theorem III 2.2 which states, in our present notation, $2\left(A_{c}-T_{c}\right)=8 t-T_{c} \geqq 0$. It follows that $r+s \leqq t$ where equality holds if and only if $C P(\alpha)$ of $T(\alpha)$ is a $C C$-support. From this inequality we have in turn $T_{c} \leqq A_{c}, 4 A_{i} \leqq 3 A_{c}$, and $T \leqq A^{*}$.

We have yet to discuss the case where $\Omega^{*}$ is flat in the direction of a side of $I A(\alpha)$. The construction $(1 / 2) T(\alpha)$, is made as before but, in this case, a side of triangle $O V_{1} V_{3}^{\prime}$ will be an edge of $(1 / 2) T(\alpha)$ and $(1 / 2) T(\alpha)$ will be a 2 -arc whose vertices are end points of this edge. In the set of values of $\alpha$ which determine $I A(\alpha)$ there will be one such that $C C(\alpha)$ of $\Omega^{*}$ is a parallelogram. When this is the case, $r+s=t$.

The next two theorems summarize the preceding remarks.

THEOREM 2.2. If $\Omega^{*}$, not a segment, is flat in the direction of a side of IA $(\alpha)$ of $\Omega^{*}, T(\alpha)$ is a 2-arc center equivalent to $\Omega^{*}$. If CC $(\alpha)$ of $\Omega^{*}$ is a parallelogram, then $T(\alpha)$ has as $C P(\alpha)$ and principal CC-support, a congruent parallelogram.

THEOREM 2.3. If $\Omega^{*}$ is not a segment and if $C P(\alpha)$ of $T(\alpha)$ is a CC-support, then $2 A_{i} / 3 \leqq T \leqq A^{*} \leqq T_{c}=A_{c}=4 A_{i} / 3$ and $A_{i} \leqq A^{*}=(1 / 2)\left(T+T_{c}\right)$. Here $2 A_{i} / 3=T$ if and only if $A_{i}=A^{*}$, and $T=A^{*}$ if and only if $A_{c}=A^{*}$.

We have shown that to each real $\alpha$ corresponds a triarc $T(\alpha)$. We note $T(\alpha \pm \pi / 3)$ is a translation of $-T(\alpha)$. If we make no distinction between $T(\alpha)$ and its reflection, we see that in general the set of $T(\alpha)$ is in one-one correspondence with intervals of $\alpha$ of length $\pi / 3$.

Finally, from the construction of Fig. 1, it is evident that we may start with any proper triarc $\Omega$, form $\Omega^{*}$, choose $I A(\alpha)$ of $\Omega^{*}$ so that one of its major diagonals corresponds to the vector joining two vertices of $\Omega$, and obtained $\Omega$ as $T(\alpha)$. From this observation and from Theorems 2.1 and 2.2, we have the following theorem.

TheOREM 2.4. Every proper triarc has a principal $C P(\alpha)$. 
3. Triarcs of minimum area. In the theorem below we find that a convex body whose area is no greater than the area of any center equivalent body is a triarc. This is only a necessary condition. Triarcs with this property are called triarcs of minimum area.

Convex bodies discussed in this section are assumed not to be segments. We continue to use the notation of Theorem 2.3.

THEOREM 3.1. In the set of bodies center equivalent to a convex body $\Omega, a$ body of minimum area is a triarc $\mathfrak{T}$. $\mathfrak{I}$ has a principal $C C$-support $\mathfrak{S}$. To $\mathfrak{T}$ corresponds a center equivalent proper triarc of minimum area which also has $\mathfrak{S}$ as principal CC-support.

Proof. By Theorem III 5.2, $\Re$ has a $C C$-support $\mathfrak{S}$. Choose $\alpha$ so that $\mathfrak{H}$ is $C P(\alpha)$ of $\Omega$.

Case I. Suppose $\mathfrak{S}$ is a parallelogram. Then $\Re$ is a flat in a direction of a side of $\mathfrak{S}$ and, by Theorem 2.2, $\mathfrak{S}$ is a principal $C C$-support of the 2 -arc $T(\alpha)$. Let the directions $\alpha_{1}$ and $\alpha_{2}$ of Theorem III 1.1 be the directions of adjacent sides of $\mathfrak{S}$. By that theorem and by Theorems 1.7 and III 1.2, we have

$$
\begin{aligned}
& T=T_{c}-\sum_{k=2}^{\infty} a_{k}, \\
& A=T_{c}-\sum_{k=2}^{\infty} \mu_{k} a_{k},
\end{aligned}
$$

and

$$
A^{*}=T_{c}-\frac{1}{2} \sum_{k=2}^{\infty} a_{k},
$$

where $1 / 2 \leqq \mu_{k} \leqq 1$ for $k=2,3, \cdots$. We conclude that $T \leqq A \leqq A^{*}$.

Case II. If $\mathfrak{S}$ is a $C$-hexagon, we have by Theorem 2.1 that $T(\alpha)$ is a 3 -arc resting on alternate vertices of $C P(\alpha)$ of $T(\alpha)$, a hexagon center equivalent to $\mathfrak{S}$. Let the $\alpha_{1}, \alpha_{2}$, and $\alpha_{3}$ of Theorem III 1.1 be the directions of consecutive sides of $\mathfrak{S}$. By that theorem and by Theorem 2.3 .

$$
\begin{aligned}
& T=T_{c}-\sum_{k=3}^{\infty} a_{k}, \\
& A=A_{c}-\sum_{k=3}^{\infty} \mu_{k} a_{k},
\end{aligned}
$$

and

$$
A^{*}=A_{c}-\frac{1}{2} \sum_{k=3}^{\infty} a_{k}
$$

where $1 / 2 \leqq \mu_{k} \leqq 1$ for $k=3,4, \cdots$. Again $T \leqq A \leqq A^{*}$. 
The inequality $A \leqq A^{*}$ is well known. The inequality $T \leqq A$ tells us the area of $\Omega$ is not less than the area of a center equivalent triarc. Now assume $\Omega$ itself has minimum area. Here we restrict the discussion to the more complicated case, Case II. Since $T=A, T_{c}=A_{c}$ and $\mu_{k}=1$ for $k=3,4, \cdots$. From the discussion following Theorem 2.1, we have $T_{c}=A_{c}$ only if $T(\alpha)$ has $\mathfrak{S}$ as principal $C C$-support. The equalities $\mu_{k}=1$ imply that $\Omega$ rests on at least one end point of each of the three major diagonals of $\mathfrak{S}$. By Theorem $1.8, \Re$ is a 3 -arc with $\mathfrak{S}$ as principal $C C$-support.

COROLlaRY 3.2. Every proper triarc of minimum area has a principal CCsupport.

CoROLlary 3.3. In the notation of the previous theorem, $2 A / 3 \leqq T \leqq A \leqq A^{*}$ $\leqq 3 A / 2$ where the first equality sign holds if $\Omega$ is an $A$-hexagon, the last equality sign holds if $\Omega$ is a triangle, and the middle equality signs holds simultaneously if $\Omega$ is a parallelogram.

The last corollary is essentially known. It follows directly from Theorem 2.3 and Corollary 1.10. The next corollary follows from the proof of Theorem 3.1 .

Corollary 3.4. A 2-arc is always a triarc of minimum area. A triarc of minimum area center equivalent to $\Omega$ is a 2-arc if and only if $\Omega$ is flat. Two center equivalent 2-arcs of minimum area have a common principal CC-support.

If we make no distinction between a convex body and its reflection, it is easy to verify that a 2 -arc $\mathfrak{T}$ of minimum area has a distinct center equivalent 2 -arc of minimum area if and only if $\mathfrak{T}$ is a 2 -arc $R S$ with a principal $C C$-support $R S T U$ such that $\mathfrak{T}$ is flat in direction $S T$ and such that $\mathfrak{T}$ does not rest on points $T$ and $U$. The second 2 -arc is then a 2 -arc $S T$ with the same $C C$-support.

The question of the uniqueness of proper 3-arcs of minimum area is much more complicated. In specific cases the description of the 3-arc often insures uniqueness. In general, from the relations given immediately above Theorem 2.2 , we see that center equivalent proper $3-\operatorname{arcs} T(\alpha)$ and $T(\beta)$ have minimum area if and only if $I A(\alpha)$ and $I A(\beta)$ of their centralization have maximum area. The latter holds only if $T(\alpha)$ and $T(\beta)$ have vertex triangles of equal area.

If, however, a proper 3-arc of minimum area is unique, it is easy to dispose of the question of whether or not there exists a center equivalent nonproper 3-arc of minimum area.

TheOREM 3.5. A proper 3-arc $\mathfrak{T}^{\prime}$, corresponding in the sense of Theorem 3.1 to a nonproper 3-arc $\mathfrak{T}^{\prime \prime}$ of minimum area, has the property that, in the direction of each of two sides of $\mathfrak{S}$, the sums of the lengths of edges of $\mathfrak{T}^{\prime}$ parallel to a side is not less than the length of the side. 
Proof. Let $\mathfrak{I}^{\prime \prime}$ be a 3-arc $L M N$ with principal $C C$-support $L M N R S T$. Let $S_{1}\left[S_{2}\right]$ be a point in which $\mathfrak{T}^{\prime \prime}$ meets $R S$ [ST]. Let the reflection of arc $S_{1} S_{2}$ in the boundary of $\mathfrak{T}^{\prime \prime}$ with respect to the center of $L M N R S T$ be an arc $M_{1} M_{2}$. Using an obvious notation it is clear that either $\mathfrak{T}^{\prime}$ or its reflection is the body $L M_{1} M_{2} N S_{1} S S_{2} L$. The edges of $\mathfrak{T}^{\prime}$ parallel, for example, to $L M$ have lengths whose sum is not less than $L M_{1}+S_{1} S=L M$.

THEOREM 3.6. If $\mathfrak{T}$ is a proper 3-arc of minimum area and $\mathfrak{S}$ is a principal $C C$-support of $\mathfrak{T}$, then in each set of three alternate sides of $\mathfrak{S}$ there is one side which meets $\mathfrak{T}$ only at an end point.

Proof. Assume the contrary. Suppose, for example, that $\mathfrak{T}$ has vertices $R, S$, and $T$, that $\mathfrak{S}$ is hexagon $R R_{2} S S_{2} T T_{2}$, and that on $\mathfrak{T}^{0}$ there is an interior point $R_{1}$ [resp., $S_{1}, T_{1}$ ] of side $R T_{2}$ [resp., $S R_{2}, T S_{2}$ ] of $\mathfrak{S}$. We lose no generality in assuming that vectors $\left[R R_{1}\right],\left[S S_{1}\right]$, and $\left[T T_{1}\right]$ sum to zero. Let $r$ [resp., $s, t]$ be the arc of $\mathfrak{I}^{0}$ from $R$ to $S_{1}$ [resp., $S$ to $T_{1}, T$ to $R_{1}$ ]. Let $R_{4}, S_{4}$, and $T_{3}$ be defined by the vector equations $\left[R_{2} R_{4}\right]=\left[S_{1} S\right]$ and $\left[S_{2} S_{4}\right]$ $=\left[T_{1} T_{3}\right]=\left[R R_{1}\right]$. We may assume $R_{1}$ is sufficiently close to $R$ to insure that $S_{1}$ is between $R_{4}$ and $S$. We define $R_{3}$ and $S_{3}$ by the equations $\left[R_{1} R_{3}\right]=\left[T T_{1}\right]$ and $\left[S S_{3}\right]=\left[R R_{1}\right]$.

Now let $\Omega$ be the 3 -arc $R_{1} S T_{3}$ whose boundary consists of segment $R_{1} R_{3}$, a translation $R_{3} S$ of $r$, segment $S S_{3}$, a translation $S_{3} T_{3}$ of $s$, segment $T_{3} T$, and $t$. It is evident that $\Re$ is center equivalent to $\mathfrak{T}$ and that the area of $\mathfrak{S}$ minus the area of $\mathfrak{T}$ equals the area of $P$-hexagon $R_{1} R_{4} S S_{4} T_{3} T_{2}$ minus the area of $\Omega$. Using Corollary III 2.3, we obtain the contradiction that $\Omega$ has less area than $\mathfrak{T}$.

From the discussion of Theorem 3.5, the following corollary is seen to hold.

CoRollary 3.7. A nonproper 3-arc LMN of minimum area has no points, other than $N$ and $L$, in common with sides $N R$ and $T L$ of a principal $C C$-support, LMNRST.

Even with the preceding theorems we are left with more possibilities than are probably necessary for the position of a proper 3-arc $L N S$ of minimum area with respect to its $C C$-support $L M N R S T$. The 3-arc $L N S$ may rest on either 5,4 , or 3 vertices of its $C C$-support, e.g.,

(i) $L, N, R, S$, and $T$. In this case $L N S$ has no points other than $L$ or $N$ in common with $L M$ and $M N$.

(ii) $L, M, N$, and $S$.

(iii) Only $L, N$, and $S$.

In the last two cases $L N S$ must have the property of Theorem 3.6. Only in the last case can $L N S$ have an associated nonproper 3-arc of minimum area.

4. Lebesgue's theorem. We conclude the chapter by giving a simple example of an application of Theorem 3.1. 
TheOREM 4.1. An orbiform of width $D$ and minimum area is a Reuleaux triangle.

Proof. Let $\Omega$ be the orbiform sought. Since orbiforms have no edges, $\Omega$ is not flat. By Theorem 3.1, $\Omega$ is a 3-arc $R S T$. If $\Omega$ is proper, $R S=S T=T R$ $=D$ and $\Omega$ is the Reuleaux triangle $R S T$. Theorem 3.5 insures that no improper solution exists. Hence $\Omega$, modulo rigid motions, is unique.

\section{Chapter V. Yamanouti triarcs}

In this chapter we establish properties of a special class of triarcs. The results are needed in Chapters VI and VIII.

1. Definitions and notation. Consider a triangle $R S T$ which has at least one altitude of length less than or equal to $E$ and whose sides $r, s$, and $t$ are greater than or equal to $E$. Let $\Gamma_{r}$ be the smaller arc of $C(R, E)$ with end points on $R S$ and $R T$. Arcs $\Gamma_{s}$ and $\Gamma_{t}$ are similarly defined. The convex hull, $\Omega_{0}$, of triangle $R S T, \Gamma_{r}, \Gamma_{s}$, and $\Gamma_{t}$ is called a Yamanouti triarc. Clearly $\Omega_{0}$ is a proper triarc of thickness $E$ and diameter max $[r, s, t]$. If the altitude on $R$ has length not less than $E$, points opposite $R$ form the segment $S T$. Otherwise these points lie on segments $S R_{1}, R_{2} T$, and an arc $R_{1} R_{2}$ of $C(R, E)$ where $S R_{1}$ and $R_{2} T$ are tangent to $C(R, E)$. Thus $\Omega_{0}$ is a 3 -arc if and only if all altitudes of $R S T$ have length less than $E$. If $r=s=t, \Omega_{0}$ is called an equilateral Yamanouti triarc.

The following theorem permits us to speak of the Yamanouti triarc associated with a given proper triarc.

THEOREM 1.1. To each proper triarc $\Omega$ of thickness $E$ there corresponds a unique Yamanouti triarc $\Omega_{0}$ imbedded in $\Omega$ and having the same vertices and same thickness.

Proof. Let $\mathfrak{S}$ be a principal $C C$-support of $\Omega$. If $\Omega$ is a proper 3-arc, let $R, S$, and $T$ be its vertices. If $\Omega$ is a 2 -arc, let $R$ and $S$ be its vertices and let $T$ be a point of $\Omega$ of maximum distance from $R S$. We note that min $[r, s, t]$ $\geqq$ thickness of $\mathfrak{S} \geqq E$. Moreover the thickness of $\triangle R S T$, the length of its shortest altitude, is less than or equal to $E$. The Yamanouti triarc $\Omega^{0}$, constructed as above, satisfies the stated conditions.

For convenience in the discussions to follow we need other definitions. If the altitudes on $R$ and $S$ have lengths less than $E$, the convex hull of $R S T$, $\Gamma_{r}$, and $\Gamma_{s}$ is called the Gothic 2 -arc $R^{*} S^{*} T$. If the altitude on $R$ has length less than $E$, the convex hull of $R S T$ and $\Gamma_{r}$ is called the semi-Gothic 2-arc $R^{*} S T$. The closure of the set of points in the semi-Gothic 2-arc $R^{*} S T$ but not in $\triangle R S T$ is called the Moorish 2-arc $R^{*} S T$. Thus a Yamanouti triarc $R S T$ is a triangle, a semi-Gothic 2-arc, a Gothic 2-arc, or a Yamanouti 3 -arc when it is formed by adjoining to $\triangle R S T$, respectively, $0,1,2$, or 3 Moorish 2-arcs. 
Let $\rho, \sigma, \tau$, and $\delta$ be respectively the arccosines of $E / r, E / s, E / t$, and $E / D$. Let $f(r)=E^{2}(\tan \rho-\rho), f(s)=E^{2}(\tan \sigma-\sigma)$, etc. Let $a$ or $a(r, s, t)$ be the area of $\triangle R S T$. Let $F(r, s, t)=f(r)+f(s)+f(t)+\pi E^{2} / 2-2 a$.

Denoting derivatives with respect to $r$ by primes, we have $f^{\prime}(r)$ $=E\left(r^{2}-E^{2}\right)^{1 / 2} / r$ and $f^{\prime \prime}(r)=E^{3} / r^{2}\left(r^{2}-E^{2}\right)^{1 / 2}$. The following theorem is seen to hold.

THEOREM 1.2. The area of a circle cap whose base is a circular arc of radius $E$ and whose peak is a distance of $x$ from the center of the circle equals $f(x) . f(x)$ is strictly increasing and strictly convex, $E \leqq x<\infty$.

THEOREM 1.3. If a capping body of $\Im$ contains more than one circle cap with a base in the same circular arc of $\Im^{0}$, these circle caps may be replaced by a single circle cap without changing the perimeter or area of the capping body.

Proof. It is sufficient to consider the case of 2 circle caps $R P S$ and $S Q T$ subtending respectively central angles of $2 \alpha$ and $2 \beta$ in a circle of radius $E$. We have $2 E(\alpha+\beta)<2 E(\tan \alpha+\tan \beta)<2 E \tan (\min [\alpha+\beta, \pi / 2])$. Since the end expressions are bounds for lengths of paths from $R$ to $T$ determined by a single circle cap, and since the middle expression is the sum of the lengths of the sides of the given caps, it is clear that these caps may be replaced by a single cap which leaves the perimeter unchanged. If the new cap subtends an angle of $2 \gamma$, we have $2 E \tan \gamma+2 E(\alpha+\beta-\gamma)=2 E(\tan \alpha+\tan \beta)$. From this we obtain the desired area relation $E^{2}(\tan \gamma-\gamma)=E^{2}(\tan \alpha-\alpha)$ $+E^{2}(\tan \beta-\beta)$.

LemмA 1.4. Let $30^{\circ} \leqq R, S, T \leqq 90^{\circ}$ and let $2 Q=f(r)+f(s)+E^{2} T-2 a$. If the altitude on $T$ has length greater than $E, Q$ is negative. Otherwise $Q$ equals the area of the Moorish 2-arc $T^{*} R S$.

Proof. The latter statement is easy to prove. In proving the former, fix $r, s$, and $t$ and denote derivatives with respect to $E$ by primes. Let $Q_{0}=2 Q / E^{2}$. Then

$$
\begin{aligned}
& Q_{0}=\tan \rho-\rho+\tan \sigma-\sigma+T-\sec \rho \sec \sigma \sin T, \\
& Q_{0}^{\prime} E=-\tan \rho-\tan \sigma+2 \sin T \sec \rho \sec \sigma, \\
& Q_{0}^{\prime} E \cos \rho \cos \sigma=2 \sin T-\sin (\rho+\sigma) \geqq 2 \sin T-1 \geqq 0 .
\end{aligned}
$$

Since $Q_{0}$ is 0 when $E$ is the length of the altitude on $T, Q_{0}$ and $Q$ are negative for smaller values of $E$.

As a direct consequence of this lemma we have the following theorem.

THEOREM 1.5. If $30^{\circ} \leqq R, S, T \leqq 90^{\circ}$, the area of the Yamanouti triarc $\Omega_{0}$ is greater than or equal to $F(r, s, t)$. Equality holds if and only if no altitude of $\triangle R S T$ has length greater than $E$. 
2. Properties involving diameter. If we fix $s, t$, and $E$ and denote derivatives with respect to $r$ by primes, we have

$$
F^{\prime}(r, s, t)=E\left(r^{2}-E^{2}\right)^{1 / 2} / r-r \cot R,
$$

and

$$
F^{\prime \prime}(r, s, t)=f^{\prime \prime}(r)+\frac{1-\cos R \sin S \sin T}{\sin R \sin S \sin T} .
$$

Thus $F(r, s, t)$ is strictly convex in $r$.

LEMma 2.1. If $r \leqq s \leqq t, F(r, s, t) \geqq F(s, s, t)$.

Proof. It is clearly sufficient to show that $F^{\prime}(r, s, t)$ is negative for $r=s \leqq t$. This holds since

$$
F^{\prime}(r, s, t)<E\left(r^{2}-E^{2}\right)^{1 / 2} / r-r \cot 60^{\circ}<0 .
$$

Lemma 2.2. If $r \leqq s \leqq t$ and if $R \geqq 30^{\circ}$, then $F(r, s, t) \geqq F(t, t, t)$.

Proof. By Lemma 2.1, it is sufficient to let $r=s$ and to show that $F(r, r, t)$ decreases with $r$. Indeed $F^{\prime}(r, r, t)=2 f^{\prime}(r)-2 r$ tan $R \leqq 2 E\left(r^{2}-E^{2}\right)^{1 / 2} / r$ $-2 r / 3^{1 / 2}<0$.

TheOREM 2.3. Let $\Omega_{0}$ be the Yamanouti triarc of thickness $E$ determined by the triangle $R S T$. Let $r \leqq s \leqq t \leqq 2 E / 3^{1 / 2}$. If $r<t, \Omega_{0}$ is not a solution of the $(D, E)$ problem described in Chapter $\mathrm{I}$.

Proof. We may assume $t=D$. Clearly $30^{\circ} \leqq R, S, T \leqq 90^{\circ}$. By Lemma 2.2, $F(r, s, t)>F(D, D, D)$. By Theorem 1.5, the area of $\Omega_{0}$ exceeds $F(D, D, D)$. But $F(D, D, D)$ is the area of an equilateral Yamanouti triarc of thickness $E$ and diameter $D$.

THEOREM 2.4. If $E \leqq r \leqq s \leqq t \leqq D \leqq 3^{1 / 2} t$ and if $R \geqq 30^{\circ}$, we have the inequality $F(r, s, t)+f(D) \geqq F(D, D, D)$. Equality holds only if $E=D$.

Proof. By Lemma 2.2, $F(r, s, t)+f(D) \geqq F(t, t, t)+f(D) \geqq 2 f(t)+f(D)$ $+\pi E^{2} / 2-2 a(t, t, t) \geqq F(t, t, D) \geqq F(D, D, D)$.

3. Properties involving perimeter. In this section we show that certain Yamanouti triarcs are not solutions of the $(C, E)$ problem described in Chapter I. These results are applied in Chapter VIII. In several of the proofs a mechanical argument is used in place of a long and tedious analytic proof.

Theorem 3.1. Consider a Gothic 2-arc $R^{*} S^{*} T$ for which $s \neq r$, or a semiGothic 2-arc $R^{*} S T$ for which the distance from $S$ to $R T$ is not less than $E$. There exists a 2-arc of the same type, the same perimeter, and smaller area, determined by $R, S$, and a point $T^{\prime}$ near $T$. 
Proof. We may assume $s<r$. Let $h$ be the distance from $T$ to $R S$. Let $\Omega$ be the 2 -arc under consideration. Let $T_{0}$ be the point of intersection of $C(R, E)$ and $C(S, E)$ nearest $T$. Let $\Omega_{0}$ be the Gothic 2 -arc $R^{*} S^{*} T_{0}$. Let $\Gamma$ be the boundary curve of $\Omega$. The reader is asked to think of $\Omega_{0}$ as a fixed structure and $\Gamma$ as a flexible, frictionless, weightless, inextensible cord which surrounds $\Omega_{0}$ and is attached to $\Omega_{0}$ at points $R$ and $S$. At the point $T$ let $\Gamma$ pass through a freely sliding ring. Let a constant gravitational field be introduced with a direction perpendicular to $R S$ and towards $T$. The ring is then allowed to slide a small distance to a new position $T^{\prime}$.

For the Gothic 2-arc $\Omega$, the formula $A=E(C-t) / 2-a$ is easily established. In the process described above, $E, C$, and $t$ remain fixed. Since $h$ increases, $a$ increases, and $A$ decreases. For the semi-Gothic 2 -arc $\Omega$, we find $A=E(C-s-t) / 2$. Here $E, C$, and $t$ are fixed and since $s$ increases, $A$ decreases.

THEOREM 3.2. If a circle capping body of either 2-arc described in Theorem 3.1 has thickness $E$, it may be subjected to a deformation which preserves thickness and perimeter and decreases area.

Proof. If the addition of a circle cap to a body increases its area by $f(x)$, its perimeter is increased by $2 f(x) / E$. Thus no change occurs in $A-E C / 2$. Hence, as in the proof of the previous theorem, it is sufficient to note that we can increase $h$ or $s$ by deforming $\Gamma$. This is done in this case by reducing the size of a circle cap and removing the resulting slack in cord $\Gamma$ by pulling out on the ring at $T$.

THEOREM 3.3. No circle capping of a Yamanouti 3-arc is a solution of the $(C, E)$ problem.

Proof. In this case we have $A=E C / 2-2 a$. We may proceed as in the previous theorem to increase $h$, increase $a$, and decrease $A$.

THEOREM 3.4. No nonequilateral Yamanouti 3-arc is a solution of the $(C, E)$ problem.

Proof. In triangle $R S T$ all altitudes have length less than $E$. We may assume $E \leqq s \leqq t \leqq r$ where $s<r$. Chord $R S$ splits the Yamanouti 3-arc $R S T$ into a Gothic 2-arc $R^{*} S^{*} T$ and a Moorish 2-arc $T^{*} R S$. We proceed by deforming these in turn.

Using the process described in the proof of Theorem 3.1, we deform the Gothic 2 -arc $R^{*} S^{*} T$ into a Gothic 2 -arc $R^{*} S^{*} T^{\prime}$. Let the boundary excluding segment $R S$ of the Moorish 2-arc $T^{*} R S$ be thought of as a cord $\Gamma$ attached to fixed points $R$ and $S$. Imagine a wheel $W$ in the plane of $R S T$ which has center $T$ and radius $E$. Then for a gravitational field perpendicular to $R S$ and away from $T, \Gamma$ has the form of a cord on which $W$ rests. $W$ meets $\Gamma$ at an arc $T_{1} T_{2}$, and $R T_{1}$ and $T_{2} S$ are tangent to this arc. Release $W$ and let it 
roll or slide along $\Gamma$ until its center reaches a point $T^{\prime \prime \prime}$ such that $T^{\prime} T^{\prime \prime \prime}$ is perpendicular to $R S$. Clearly $T^{\prime \prime \prime}$ is closer to $R S$ than $T$. Let $T^{\prime \prime}$ be the point on $T^{\prime} T^{\prime \prime \prime}$ such that $T T^{\prime \prime}$ is parallel to $R S$. Move $W$ so its center is at $T^{\prime \prime}$. There is now slack in $\Gamma$. Introduce between $W$ and $\Gamma$ a block in the form of a circle cap $X Y Z$ whose base is on the rim of $W$ and which is just large enough to restore tautness in $\Gamma$. Now $\Gamma$ has the form of a segment $R T_{1}^{\prime \prime}$, an arc $T_{1}^{\prime \prime} X$, segments $X Y$ and $Y Z$, an arc $Z T_{2}^{\prime \prime}$, and a segment $T_{2}^{\prime \prime} S$. We have deformed the Moorish 2-arc $T^{*} R S$ into a circle capping body of the Moorish 2-arc $T^{\prime \prime *} R S$. Each has as perimeter, $C^{\prime \prime}$, the length of $R S$ plus the length of $\Gamma$. Elementary computations establish that they have the same area, $E\left(C^{\prime \prime}-t\right) / 2-a$.

When the deformations described for the Gothic 2 -arc $R^{*} S^{*} T$ and the Moorish 2-arc $T^{*} R S$ are carried out simultaneously, the Yamanouti 3-arc is clearly deformed into a convex body $R T_{1}^{\prime \prime} X Y Z T_{2}^{\prime \prime} S T^{\prime}$ which has the same thickness, same perimeter, and less area.

The results of this section may be summarized as follows.

THEOREM 3.5. If a solution of the $(C, E)$ problem is a Yamanouti triarc $\Re$, then $\Omega$ is either a triangle or an equilateral Yamanouti 3-arc. No circle capping body of a Yamanouti triarc is a solution of the $(C, E)$ problem.

\section{Chapter Vi. The $(D, E)$ Problem}

In this chapter we solve the problem of finding the body of minimum area which has a given diameter $D$ and thickness $E$. We denote a solution by $\Re(D, E)$ and its area by $A(D, E)$. Two solutions are not considered distinct if one is obtained from the other by a rigid motion. The notation $(D, E)$ body refers to a convex body of diameter $D$ and thickness $E$.

1. Summary of results. In the following theorem, Case (i) was proved by Kubota [6]. Case (iii), proved by Lebesgue [8,9], was considered in §IV 4. The proof of Case (ii) is given in the next section.

Theorem 1.1. With the exception of Case (i) below, $\Re(D, E)$ is unique. If we have:

(i) $0 \leqq 2 E / 3^{1 / 2} \leqq D, ~ \Re(D, E)$ is a triangle of base $D$ and height $E$, and $A(D, E)=D E / 2$.

(ii) $0<E<D<2 E / 3^{1 / 2}, \Re(D, E)$ is an equilateral Yamanouti triarc, and $A(D, E)=\left(\pi E^{2}-3^{1 / 2} D^{2}\right) / 2+3 E^{2}(\tan \delta-\delta)$, where $\delta=\cos ^{-1}(E / D)$.

(iii) $0<E=D, \quad \Re(D, E)$ is a Reuleaux triangle and $A(D, E)$ $=\left(\pi-3^{1 / 2}\right) D^{2} / 2$.

If we set $x=E / D$ and $y=A(D, E) / D^{2}$, we have $y=x / 2,0 \leqq x \leqq 3^{1 / 2} / 2$. For $3^{1 / 2} / 2 \leqq x \leqq 1$, we have

$$
\begin{aligned}
y & =\left(\pi x^{2}-3^{1 / 2}\right) / 2+3 x^{2}(\tan \delta-\delta), \\
y^{\prime} & =x(\pi-6 \delta)+3\left(1-x^{2}\right)^{1 / 2}
\end{aligned}
$$


and

$$
y^{\prime \prime}=\pi-6 \delta+3 x /\left(1-x^{2}\right)^{1 / 2},
$$

where $\delta=\cos ^{-1} x$. Since $\delta$ decreases from $\pi / 6$ to 0 , we see that $y$ is strictly increasing and strictly convex and $y^{\prime}$ increases from $3 / 2$ to $\pi$.

Theorem 1.2. For a convex body of area $A$, diameter $D$, and thickness $E$ the following simple sharp inequalities hold:

(i) $2 A \geqq D E$.

(ii) $2 A \geqq 3 D E-3^{1 / 2} D^{2}$.

(iii) $2 A \geqq \pi E^{2}-3^{1 / 2} D^{2}$.

The first inequality was proved by Kubota. The second inequality follows from the relation of the graph of $y=f(x)$ to its supporting line of slope $3 / 2$ at the point $\left(3^{1 / 2} / 2,3^{1 / 2} / 4\right)$. The third inequality follows from the ex pression for $y$ and the inequality $\tan \delta-\delta \geqq 0$. The third inequality is uniformly better than the inequality $2 A \geqq D\left(2 \pi E-\pi D-3^{1 / 2} D\right)$ determined by constructing the tangent to $y=f(x)$ at the point $\left(1, \pi / 2-3^{1 / 2} / 2\right)$. The domains for which the inequalities are particularly good are, respectively, $0 \leqq x \leqq 3^{1 / 2} / 2,3^{1 / 2} / 2 \leqq x \leqq 3 / \pi$, and $3 / \pi \leqq x \leqq 1$.

2. The case $0<E<D<2 E / 3^{1 / 2}$. By Blaschke's Selection Theorem, we know $\Re(D, E)$ exists. We assume $\Re(D, E)$ is not an equilateral Yamanouti triarc and show that this leads to a contradiction.

In this section we denote $\Re(D, E)$ by $\Re$ and use the notation of $\S \mathrm{V} 1$. We first note that, since center equivalent bodies have the same diameter and thickness, the following lemma follows from Theorem IV 3.1.

Lemma 2.1. $\Omega$ is a triarc. We may assume $\Omega$ is a proper 3-arc RST or a 2-arc $R S$. $\Omega$ has a principal $C C$-support, $\mathfrak{S}$.

If $\Re$ is a 2 -arc, let $T$ be a point of $\Re$ whose distance from $R S$ is maximum. We lose no generality by assuming $E=1$. We proceed as in $\S \mathrm{V} 1$. From $1 \leqq r, s, t \leqq D<2 / 3^{1 / 2}$, we have $0 \leqq \rho, \sigma, \tau \leqq \delta<\pi / 6$ and $51^{\circ}<\cos ^{-1} 5 / 8<R, S, T$ $<\cos ^{-1} 1 / 3<71^{\circ}$. Let $\Omega_{1}$ be the Yamanouti triarc associated with $\Omega$. It is contained in $\Omega$, its thickness is 1 , and its diameter is max $[r, s, t]$.

Lemma 2.2. At most one altitude of triangle RST has length greater than or equal to 1.

Proof. If, say, $R P$ and $S Q$ are altitudes of length not less than one, the least possible values of $r$ and $s$ occur when $R P=S Q=1$. Hence, since $t<2 / 3^{1 / 2}$, we have the contradiction that $r$ and $s$ exceed $2 / 3^{1 / 2}$.

We may assume that the altitude on $T$ has maximum length since, if $\Omega$ is the 2 -arc $R S$, the altitude on $T$ must be no less than 1 and must therefore have maximum length.

Lemma 2.3. There is a point $P$ not in $\Omega_{1}$ such that $\Re$ is the convex hull of 
$P$ and $\Omega_{1}$, and such that $P$ is a distance of $D$ from a vertex, say $R$, of $\Omega$. The diameter of $\Omega_{1}$ is less than $D$.

Proof. By Theorem V 3.5, $\Omega_{1}$ is identical with $\Omega$ only if it is equilateral and of diameter $D$. Since this contradicts our assumption with regard to $\Omega$, we have that $\Omega_{1}$ is a proper subset of $\Omega$. This, in turn, is a contradiction if $\Omega_{1}$ has diameter $D$. Hence $r, s, t<D$ and $\Omega$ contains a point $P$ distinct from $R, S$, or $T$ such that $P$ is the endpoint of a chord of length $D$. We have an immediate contradiction unless $\Omega$ is the convex hull of $\Omega_{1}$ and $P$ since, by the

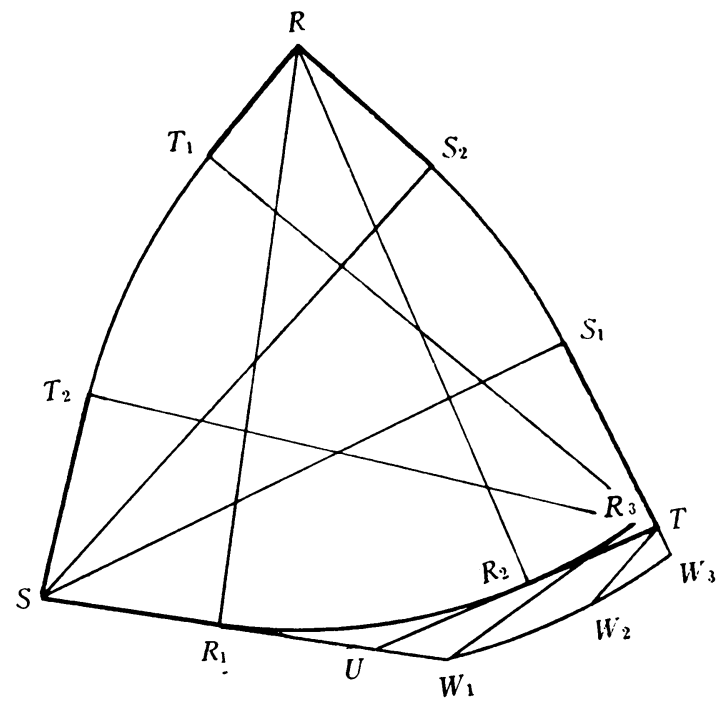

FIG. 2

previous lemma, this convex hull is necessarily a $(D, E)$ body contained in $\Omega$. Finally, if the altitude of triangle $R S T$ on $T$ has a length not less than one, the assumption that $P$ is opposite $T$ leads to a contradiction. In this case $\Omega_{1}$ is a Yamanouti 3-arc and $P$ may be displaced on the circle $C(T, D)$ to a position $P_{0}$ outside $\mathfrak{S}$, and the convex hull of $P_{0}$ and $\Omega_{1}$ is a $(D, E)$ body of smaller area than $\Re$. We may therefore assume $P$ is opposite, say, $R$.

In the discussion that follows we use the phrase "inside [outside] the line $t$ " to mean "in the one of the two open half-planes determined by $t$ whose closure has [does not have] $\Omega_{1}$ as a subset." The principal constructions are shown in Fig. 2.

Let $\Omega_{1}$ be the triarc $R T_{1} T_{2} S R_{1} R_{2} T S_{1} S_{2} R$. Let $S R_{1}$ meet $T R_{2}$ at $U$. The point $P$ of Lemma 2.3 cannot lie outside both $S U$ and $T U$ for, if such were the case $P$ could be displaced on $C(R, D)$ to obtain a $(D, E)$ body of smaller area than $\Re$. We may assume $P$ lies inside $S U$.

It follows that $P$ lies outside $T U$, for if $P$ lies inside or on $T U$, we have 
$A(D, E)$ equal to the area of $\Omega_{1}$ plus the area $f(D)$ of a circle cap. From Theorems V 1.5 and V 2.4 we have

$$
A(D, E) \geqq F(r, s, t)+f(D)>F(D, D, D),
$$

and this, as in the proof of Theorem V 2.3, is a contradiction.

Let $W_{1}, W_{2}$, and $W_{3}$ be points on $C(R, D)$ such that $W_{3}$ is on $S_{1} T$ extended, $T W_{2}$ is parallel to $R T_{1}$, and $W_{1}$ is $U$ [resp., is on $T U$, is on $S U$ extended] if $U$ lies on [resp., outside, inside] the circle $C(R, D)$. These three points are distinct and have the ordering given by their subscripts. Since $P$ is in $\mathfrak{S}, P$ lies on the arc $W_{1} W_{2}$.

Let $V$ be the point on arc $R_{1} R_{2}$ of $C(R, 1)$ such that $P V$ is tangent to this arc. Let $\theta$ be the angle $R_{2} R V$ and let $\epsilon$ be the angle $T R P$. We note that $\epsilon=\theta+\sigma-\delta$. Since triangles $P R V, T R P, T R R_{2}$, and sector $R_{2} R V$ have respectively the areas $(1 / 2)\left(D^{2}-1\right)^{1 / 2},(1 / 2) s D \sin \epsilon,(1 / 2)\left(s^{2}-1\right)^{1 / 2}$, and $(1 / 2) \theta$, the area of $\Omega$ exceeds the area of $\Omega_{1}$ by $(1 / 2)\left(s D \sin \epsilon+\left(D^{2}-1\right)^{1 / 2}\right.$ $\left.-\theta-\left(s^{2}-1\right)^{1 / 2}\right)$. We denote the expression in parentheses by $X$. With $r, s, t$, and $D$ fixed, $X$ is a function of $\epsilon$. Denoting derivatives with respect to $\epsilon$ by primes we have $X^{\prime}=s D \cos \epsilon-1$ and $X^{\prime \prime}=-s D \sin \epsilon$. Thus $X$ is a concave function and since a contradiction results unless $X$ has minimum value, $\epsilon$ has one of the values determined by having $P$ coincident with $W_{1}$ or $W_{2}$ We denote these values by $\epsilon_{1}$ and $\epsilon_{2}$ respectively.

Consider first the case $X\left(\epsilon_{2}\right) \leqq X\left(\epsilon_{1}\right)$. We may assume $P=W_{2}$. Since a displacement of $P$ into the arc $W_{2} W_{3}$ determines a value $\epsilon$ such that $\epsilon<\epsilon_{2}<\epsilon_{1}$ and $X(\epsilon)<X\left(\epsilon_{2}\right)$, we obtain as a contradiction the existence of a $(D, E)$ body of smaller area than $\Omega$.

We are left only with the possibility $X\left(\epsilon_{1}\right)<X\left(\epsilon_{2}\right)$ and $P=W_{1}$. This holds only if $X^{\prime}<0$ for $\epsilon=\epsilon_{1}$. Hence $\cos \epsilon<1 / s D$. Since the case where $P$ lies inside or on $T U$ has already been eliminated, we have that $W_{1}$ lies on $S R_{1}$ extended. Let $R_{3}$ be the point on $C(R, 1)$ such that $W_{1} R_{3}$ is tangent to $C(R, 1)$ and $R_{2}$ is between $R_{1}$ and $R_{3}$.

Since $\cos T R W_{1}<1 / s D \leqq 1 / D<1 / s$, angle $T R W_{1}>\delta>\sigma$. This inequality implies that $R_{2}$ and $R_{3}$ lie in the interior of triangle $W_{1} T R$. Let $W_{1} R_{2}$ and $T R_{3}$ meet at $Q_{1}$ and let $W_{1} R_{3}$ and $T R_{2}$ meet at $Q_{2}$. Trivially, the area of circle cap $R_{2} Q_{2} R_{3}$ is not greater than the area of triangle $R_{2} Q_{2} R_{3}$ and the latter, by Lemma III 2.5, is not greater than the area of triangle $Q_{2} W_{1} T$. Hence $X / 2$ is not less than the sum of the areas of circle caps $R_{2} Q_{2} R_{3}, R_{1} U R_{2}$, and triangle $U W_{1} Q_{2}$. Since this sum is the area, $f(D)$, of circle cap $R_{1} W_{1} R_{3}$, we again have the inequality $\left({ }^{*}\right)$, from which as before we may derive a contradiction.

This completes the proof of Theorem 1.1.

\section{Chapter VII. Minimum area Under imbedding Conditions}

Results given in this chapter are, in some cases, analogous to those obtained by Favard [2]. The latter sought for his purposes to replace a plane 
body by another of equal area and larger perimeter, where these bodies were subject to some imbedding condition. Others have used ellipses as they are used below in discussing length-preserving deformations of convex curves. We refer to the ellipse with foci $P$ and $Q$ which has a major axis of length $\lambda$ as the ellipse $(P, Q, \lambda)$.

Consider a convex body $\Omega_{1}$ in which a convex body $\Im$ is imbedded [resp., which is imbedded in $\Im$ ]. If there exists a convex body $\Omega_{2}$ of the same perimeter and less area in which $\Im$ is also imbedded [resp., which is also imbedded in $\Im$ ], we say that $\Omega_{1}$ can be shrunk or, more explicitly, that $\Omega_{1}$ can be shrunk over $\Im$ into $\Omega_{2}$ [resp., under $\Im$ into $\Omega_{2}$ ].

1. A fundamental lemma. The lemma to which we here refer is Lemma 1.3.

In the following discussion, $\&$ represents a triangular body $R S T$ where angle $T R S \leqq$ angle $R S T$. However the results are seen to hold if $\&$ is the unbounded convex set whose boundary has the following form. Let $T_{1} R S T_{2}$ be a convex nondegenerate quadrilateral such that the ray from $R$ through $T_{1}$ does not meet the ray from $S$ through $T_{2}$ and such that angle $T_{1} R S$ $\leqq$ angle $R S T_{2}$. Let $\mathfrak{R}^{0}$ be composed of these two rays and of the segment $R S$.

By a standard triangle in $R S T$, we mean a triangle with base $R S$ whose vertex lies on $S T$ or, when $T R S=R S T$ only, on $R T$.

LEMMA 1.1. If $P$ is in $\mathfrak{R}$ and $R S P$ is not a standard triangle, we may shrink $R S P$ under $\&$.

Proof. Let ellipse $(R, S, R P+P S)$ meet $S T$ at $Q$. We may shrink $R S P$ into $R S Q$.

Lemma 1.2. If $R S P_{1} P_{2}$ is a convex quadrilateral in $\mathfrak{R}$, we may shrink $R S P_{1} P_{2}$ into a standard triangle.

Proof. Suppose, first, $P_{1}$ lies on $S T$. By Lemma 1.1 and the angle inequality $T R P_{1}<T R S \leqq R S T<R P_{1} T$, we may shrink $R P_{1} P_{2}$ under $R P_{1} T$ into $R P_{1} Q$ where $Q$ is on $P_{1} T$. This shrinks $R S P_{1} P_{2}$ into $R S Q$.

Suppose, next, that $P_{2}$ is on $R T$. Shrink $P_{2} S P_{1}$ under $P_{2} S T$ into $P_{2} S Q$, a standard triangle in $P_{2} S T$. If $Q$ is on $S T$, we may treat $R S Q P_{2}$ as in the first case. If $Q$ is on $P_{2} T$, we apply Lemma 1.1 to $R S Q$.

Finally, if neither $P_{1}$ nor $P_{2}$ is on $\mathbb{R}^{0}$, let $R P_{2}$ meet $S T$ at $T_{1}$. By the previous case, we may shrink $R S P_{1} P_{2}$ into a standard triangle in $R S T_{1}$. This triangle is also standard in $R S T$.

Lemma 1.3. If $\Omega$ is a convex body, not a standard triangle, in $\Omega$ and if $\Omega$ has $R S$ as an edge, then $\Re$ can be shrunk into a standard triangle in $\&$.

Proof. The case where $\Omega$ is a convex polygon may be easily established by induction. Assume $\Omega$ is not a polygon. Let $P$ be a point of $\AA^{0}$ not on a supporting line of $\Omega$ through $R$ or $S$. Let a supporting line of $\Omega$ at $P$ meet $R T$ at $R_{1}$ and $S T$ at $S_{1}$. Let $\mathfrak{S}$ [resp., $\Im$ ] be the intersection of $\Re$ and triangle 
$R P R_{1}$ [resp., $P S S_{1}$ ]. By expressing $\mathfrak{S}$ as the limit of a polygon sequence we easily establish the existence in $R P R_{1}$ of a standard triangle $R P R_{2}$ with a perimeter equal to that of $\mathfrak{S}$ and with an area no greater than that of $\mathfrak{S}$. There corresponds, in the same way, to $\Im$ a standard triangle $P S S_{2}$ in $P S S_{1}$. The polygon $R S S_{2} P R_{2}$ may now be shrunk into a standard triangle.

2. Capping bodies. We use here terminology and notation introduced in Chapter II.

THEOREM 2.1. In the set of all convex bodies which contain a convex body $\Im$ and which have a given perimeter $C, a$ body $\Omega$ of minimum area is a capping body of $\Im$.

Proof. If $\Im$ is a segment, so is $\Re$. Assume $\Im$ has inner points and consider a body $\Omega$ of perimeter $C$ containing $\Im$. Let $t$ be a line of support of $\Im$ but not of $\Omega$. Let $t$ meet $\Omega^{0}$ at points $P_{1}$ and $P_{2}$. At these points let $t_{1}$ and $t_{2}$ respectively be supporting lines of $\Omega$. Let $\pi$ [resp., $\pi_{1}, \pi_{2}$ ] be the closed half-plane determined by $t$ [resp., $t_{1}, t_{2}$ ] which does not contain $\Im$ [resp., which contains $\Re]$. Let $\&$ be the intersection of these three half planes. By Lemma 1.3, $\& \cap \Omega$ is a standard triangle in $\&$. This clearly implies that each component of the subset of $\Omega^{0}$ not in $\Im^{0}$ is a polygonal line and that no edge of $\Omega$ may be separated from $\Im$ by a supporting line of $\Im$. Hence each such component consists of exactly two segments, the sides of a cap.

It is evident that if the following theorem did not hold, we could displace $Q$ on ellipse $(P, R, P Q+Q R)$ to obtain a contradiction.

THEOREM 2.2. For a cap $P Q R$ in the body $\Omega$ of the previous theorem either $P Q$ or $Q R$ is an extreme supporting line of $\Im$. If, say, $P Q \leqq Q R$ then either at $R$ there is no supporting line which separates $Q$ from $\Im$ or at $P$ there is no supporting line of $\Im$ which fails to meet $R Q$.

In the following theorem consider again a cap $P Q R$ in the body $\Omega$ of minimum area. Let the extreme supporting lines of $\Im$ at $R$ meet $P Q$ at $P_{1}$ and $Q_{1}$ so that on $P Q$ we have in order the points $P, P_{1}, Q$, and $Q_{1}$. We assume that $P_{1}, Q$, and $Q_{1}$ are distinct.

Theorem 2.3. Cap $P Q R$ has the property $P_{1} Q_{1} \leqq R Q_{1}$.

Proof. Assume the theorem false. We note $P_{1}$ is not in $\Im$ since $P$ is given as an end point of cap $P Q R$. We have $P_{1} Q \leqq R Q$ for otherwise we obtain a contradiction by displacing $Q$ on ellipse $\left(P_{1}, R, P_{1} Q+Q R\right)$. Let $Q_{2}$ be the reflection of $Q$ with respect to the perpendicular bisector of $R P_{1}$. In $\AA^{0}$ replace segments $P_{1} Q$ and $Q R$ by segments $P_{1} Q_{2}$ and $Q_{2} R$. The resulting convex body contradicts Theorem 2.1 .

3. Applications to the $(C, E)$ problem. For the statement of this problem and for some of the notation used below, see the introduction of Chapter VIII. 
Consider a convex body $\Im$ of thickness $E$ and, strictly containing $\Im$, a convex body $\Omega$ of perimeter $C$ and thickness $E$. If we may shrink $\Omega$ over $\Im$ into $\Omega_{1}$, it is clear $\Omega_{1}$ has a thickness no less than $E$ and may therefore be shrunk (not necessarily over $\Im$ ) into a $(C, E)$ body. Thus $\Re$ is not $\Re(C, E)$. By this reasoning we establish the following extensions of theorems in the previous section.

THEOREM 3.1. If $\Re$ is not a capping body of $\Im, \Re$ is not $\Re(C, E)$.

THEOREM 3.2. If the caps in $\Omega$ do not have the property of Theorem 2.2, $\Omega$ is not $\Re(C, E)$.

THEOREM 3.3. If the caps in $\Re$ do not have the property of Theorem 2.3, $\Re$ is not $\Re(C, E)$.

On the boundary of a body $\Omega(C, E)$ consider a point $P$ which is an end point of no major chord of length $E$. Moreover, if $P$ is interior to an edge of $\Omega(C, E)$, assume no point of this edge is an end point of a major chord of length $E$. Let $t$ be a supporting line at $P$. Let $s$ be the chord of $\Re(C, E)$ parallel to $t$ and at minimum distance from $t$ such that $s$ contains an end point of a major chord of length $E$. Let $\Omega_{t}$ be the simple cutting body of $\Omega(C, E)$, determined by $s$, which is disjoint from $P$. By Theorem 3.1, $\Omega(C, E)$ is a capping body of $\Omega_{t}$ and the closure of the set of points in the former body but not in the latter is a triangle with $s$ as a base and with a vertex on $t$. Thus a contradiction results unless the following theorems hold.

THEOREM 3.4. An extreme point of $\Re(C, E)$ is either a corner of $\Omega(C, E)$, the intersection of two edges of that body, or else an end point of a major chord of length $E$.

THEOREM 3.5. On every edge of $\Re(C, E)$ there is at least one point which is an end point of a major chord of length $E$.

4. Other bodies of extremal area. The theorem below supplements Theorem 2.1.

THEOREM 4.1. In the set of all convex bodies which are contained in a convex body $\Im$ and which have a given perimeter $C$, a body of minimum area is a cutting body of $\Im$.

Proof. Let $\Omega$ be a body of minimum area and let $\Gamma$ be a component of the set of points found in $\Re^{0}$ but not in $\Im^{0}$. If $\Gamma$ consists of two or more line segments, we can displace a corner, as in Lemma 1.1, to establish a contradiction. Assume $\Gamma$ contains a point of accumulation $P$ of extreme points of $\Omega$. We may select points $R$ and $S$ in $\AA^{0}$ sufficiently close to $P$ to insure that supporting lines of $\Omega$ at $R$ and $S$ meet at a point $T$ inside $\Im^{0}$. By Lemma 1.3, the subset of $\Omega$ in $R S T$ is a standard triangle in $R S T$. This contradicts our 
choice of $P$. We are forced to conclude that $\Gamma$ is a segment whose closure is a chord of $\Im$.

A convex body $\Re$ of given perimeter, either contained in or containing a given body $\Im$, which has maximum area, has the property that points in $\AA^{0}$ but not in $\Im^{0}$ form circular arcs. This and other properties of $\Omega$ may be established in a manner similar to that used for finding properties of bodies of minimum area. If one thinks of $\Re$ as the cross section of a balloon, these properties are intuitively obvious.

\section{Chapter ViII. The $(C, E)$ problem}

In this chapter we solve the problem of finding the body of minimum area which has a given perimeter $C$ and thickness $E$. We denote a solution by $\Re(C, E)$ and its area by $A(C, E)$. The notation $(C, E)$ body refers to a convex body of perimeter $C$ and thickness $E$.

1. Summary of results. In the following theorem, Case (i) was proved by Yamanouti [10] who also correctly conjectured the solution for Case (ii). Case (iii), proved by Lebesgue [8,9], was considered in §IV 4. The proof of Case (ii) is given in the next section.

Theorem 1.1. $\Re(C, E)$ is uniquely determined by $C$ and $E$. When:

(i) $0 \leqq 2\left(3^{1 / 2}\right) E \leqq C, \Re(C, E)$ is a triangle two of whose altitudes have length $E$ and $A(C, E)$ is the middle root of the equation

$$
128 C x^{3}-16 E\left(5 C^{2}+E^{2}\right) x^{2}+16 E^{2} C^{3} x-E^{3} C^{4}=0,
$$

(ii) $0<\pi E<C<2\left(3^{1 / 2}\right) E, \Omega(C, E)$ is an equilateral Yamanouti triarc and $2 A(C, E)=E\left(C-3^{1 / 2} E \sec ^{2} \gamma\right)$ where tan $\gamma-\gamma=(C-\pi E) / 6 E$,

(iii) $0<\pi E=C, \Re(C, E)$ is a Reuleaux triangle and $2 A(C, E)=E^{2}\left(\pi-3^{1 / 2}\right)$.

We now consider the graph of $y=f(x)$ where $x=C / E$ and $y=A / E^{2}$.

For $\pi \leqq x \leqq 2\left(3^{1 / 2}\right)$, we have the equations $x=\pi+6(\tan \gamma-\gamma)$ and $y$ $=\left(x-3^{1 / 2} \sec ^{2} \gamma\right) / 2$ where the parameter $\gamma$ has the range $0 \leqq \gamma \leqq \pi / 6$. We find that $y$ is a decreasing convex function of $x$ and $d y / d x$ increases from $-\infty$ to $-1 / 6$.

Let $t=4 y / x$. For $2\left(3^{1 / 2}\right) \leqq x<\infty$, we have $x=t /(1-t)(2 t-1)^{1 / 2}$ where the parameter $t$ increases from $2 / 3$ to 1 . In this interval, $y$ is an increasing concave function of $x$.

The equations of the extreme supporting lines of the graph at $\left(2\left(3^{1 / 2}\right), 1 / 3^{1 / 2}\right)$ are $6 y=-x+4\left(3^{1 / 2}\right)$ and $4 y=x-2 / 3^{1 / 2}$. From these we obtain the first two inequalities in the theorem below. The second inequality is known and was proved by Kawai [5]. For the interval $2\left(3^{1 / 2}\right) \leqq x<\infty$, Yamanouti [10] has the improved bound $4 y \geqq x-\left(1+4 / x^{2}\right)^{1 / 2}$. Improved bounds can be found for values of $x$ near $\pi$ in the form $2 y \geqq x$ $-3^{1 / 2}-3^{1 / 2}((x-\pi) / 6)^{1 / n}$.

The last inequality in the theorem below is justified by observing that 
for $\pi \leqq x<\infty$, the minimum value of $t$ is $2 / 3$. In each of the inequalities, equality holds only for an equilateral triangle.

ThEOREM 1.2. For a convex body of perimeter $C$, thickness $E$, and area $A$, the following simple sharp inequalities hold:

(i) $6 A \geqq 4 E^{2} 3^{1 / 2}-C E$,

(ii) $4 A \geqq C E-2 E^{2} / 3^{1 / 2}$,

(iii) $6 A \geqq C E$.

2. The case $0<\pi E<C<2\left(3^{1 / 2}\right) E$. By Blaschke's Selection Theorem, we know $\Re(C, E)$ exists. We assume $\Re(C, E)$ is not an equilateral Yamanouti triarc, and show that this leads to a contradiction. In the remainder of the section we denote $\Omega(C, E)$ by $\Omega$.

Since center equivalent bodies have the same perimeter and thickness, the following lemma follows from Theorem IV 3.1.

Lemma 2.1. $\Omega$ is a triarc. We may assume $\Omega$ is a proper 3-arc RST or a 2-arc. $\Re$ has a principal CC-support, $\mathfrak{S}$.

If $\Omega$ is a 2 -arc $R S$ [resp., $S T, T R$ ] let $T$ [resp., $R, S$ ] be an extreme point of $\Omega$ whose distance from $R S$ [resp., $S T, T R$ ] is maximum. We lose no generality by assuming $E=1$. If no altitude of $\triangle R S T$ has length less than 1, we have the contradiction that the perimeter of the triangle, and hence $C$, is not less than $2\left(3^{1 / 2}\right)$. Thus, associated with $\Omega$ there is a Yamanouti triarc $\Omega_{1}$ and, by Theorem VII 3.1, we have the following lemma.

Lemma 2.2. $\Omega$ is either $\Omega_{1}$ or a capping body of $\Omega_{1} . \Omega$ is not a triangle.

LEMma 2.3. Each angle of the triangle RST is less than $84^{\circ}$ and greater than $45^{\circ}$.

Proof. Assume, say, $R \geqq 84^{\circ}$. Then the perimeter of $\Omega_{1}$ is not less than the perimeter of a sector of radius 1 and central angle $R$. Hence we have the contradiction $C \geqq 2+R>2\left(3^{1 / 2}\right)$. Assume now $R \leqq 45^{\circ}$. For $s$ and $t$ fixed, the perimeter $\lambda$ of triangle $R S T$ is least if $r=1$ and $R=45^{\circ}$. Under these conditions $\lambda$ is least when $|S-T|$ is maximum. Since $|S-T|<84^{\circ}-51^{\circ}$, $C>\lambda>1+2^{1 / 2} \sin 84^{\circ}+2^{1 / 2} \sin 51^{\circ}>2\left(3^{1 / 2}\right)$, a contradiction.

We have assumed $\Omega$ is not an equilateral Yamanouti triarc. By Theorem V 3.5, $\Omega$ is neither $\Omega_{1}$ nor a circle capping of $\Omega_{1}$. Let $P Q Z$ be a cap in $\Omega$ which is not a circle cap. It is clear that at least one of the points $P$ or $Z$ is a vertex of $\triangle R S T$. If both $P$ and $Z$ are vertices and $Y$ is the third vertex, we cannot have $P Y=Z Y=1$ for, in that case, since $Q$ lies in $\mathfrak{S}$, we have $Q$ a vertex of $\mathfrak{S}$ and $P Q Z$ is a circle cap of $\Omega_{1}$. We may assume $Z=T$, that the distance from $T$ to the vertex of $\triangle R S T$ opposite $Q$ is greater than 1 , and that $s \leqq r$.

Let $r^{\prime}, s^{\prime}$, and $t^{\prime}$ be respectively the lengths of the altitudes perpendicular to $r, s$, and $t$. We have $t^{\prime} \leqq 1$ for otherwise the edge $Q T$ of $\Omega$ contradicts 
Theorem VII 3.5. The boundary points of $\Omega_{1}$ opposite $T$ consist of an arc $T_{1} T_{2}$ of $C(T, 1)$ and of segments $R T_{1}$ and $T_{2} S$ tangent to $C(T, 1)$. If $r^{\prime} \leqq 1\left[s^{\prime} \leqq 1\right]$, let points $R_{1}$ and $R_{2}\left[S_{1}\right.$ and $\left.S_{2}\right]$ be similarly defined.

\section{Lemma 2.4. $Q$ is not opposite $S$.}

Proof. Assume the contrary. If $s^{\prime} \geqq 1, R T$ is an edge of $\Omega_{1}, P=R$, and since $Q$ lies in $\mathfrak{S}$, we have a contradiction from Theorem VII 3.2. Hence $s^{\prime}<1$. $P$ lies on arc $S_{1} S_{2}$ for otherwise $P=R$ and we again have a contradiction from Theorem VII 3.2. Let $T S_{1}$ extended meet $P Q$ at $P_{1}$ and let the other extreme supporting line of $\Omega_{1}$ at $T$ meet $P Q$ extended at $Q_{1}$. We wish to use Theorem VII 3.3 to derive a contradiction. It remains to show angle $Q_{1} P_{1} T<$ angle $P_{1} T Q_{1}$. We assume the least favorable case, $P=S_{2}$ and $Q_{1} T$ parallel to $R T_{1}$. (This limiting position of $Q_{1} T$ is determined by the limiting position of parallel sides of $\mathfrak{S}$.) Let $S S_{1}$ meet $T T_{1}$ at $V$ and $T R$ at $W$.

In the notation of $\S \mathrm{V} 1$, angle $Q_{1} P_{1} T=S_{2} S S_{1}=S-\tau-\rho$ and angle $P_{1} T Q_{1}$ $=S V T_{1}=V S T+S T V=T-\sigma+\rho$. For fixed $r$ and $s$, a decrease in $t$ increases $S-\tau-\rho$ and decreases $T-\sigma+\rho$. We may assume we have minimum $t$ and hence either $t=1$ or $t^{\prime}=1$. First, if $t=1, S-\tau-\rho=S-\rho \leqq R-\sigma<S W R-\sigma$ $=T+\rho-\sigma$. Finally, if $t^{\prime}=1, T=\sigma+\rho, \rho=90^{\circ}-S$, and the problem reduces to that of showing $4 S<270^{\circ}+\tau$. This holds since, by Lemma $2.3,2 S \leqq R+S$ $=\pi-T<135^{\circ}$.

By the previous lemma there is no cap of $\Omega_{1}$ with peak opposite $S$ and with $T$ as an end point. In the following lemma we continue, of course, to assume $s \leqq r$.

Lemma 2.5. $Q$ is not opposite $R$.

Proof. Assume the contrary. We have analogously with the proof of Lemma 2.4 a contradiction from Theorem VII 3.2 unless $r^{\prime}<1$ and $P$ lies on the arc $R_{1} R_{2}$. Let $T R_{2}$ meet $P Q$ at $P_{1}$ and let the other extreme supporting line to $\Omega_{1}$ at $T$ meet $P Q$ extended at $Q_{1}$. We have a contradiction from Theorem VII 3.3 if we can show angle $Q_{1} P_{1} T<$ angle $Q_{1} T P_{1}$. We assume the least favorable case, $P=R_{1}$. If $s^{\prime} \geqq 1, T Q_{1}$ is an extension of $R T$ and we have the angle inequality $Q_{1} P_{1} T=R_{2} R R_{1} \leqq R<\pi / 2 \leqq Q_{1} T P_{1}$. If $s^{\prime}<1, T Q_{1}$ is an extension of $S_{1} T$. Let $R R_{2}$ meet $S S_{1}$ at $U$. Since $U S<1 \leqq R S$, we have the inequality $Q_{1} P_{1} T \leqq U R S \angle S U R=Q_{1} T P_{1}$.

Lemmas 2.4 and 2.5 show that the cap $P Q T$ cannot exist. This completes the proof of Theorem 1.1.

\section{Chapter IX. The $(C, D)$ problem}

In this chapter we give some properties of a convex body of minimum area, say $\Re(C, D)$, which has a given perimeter $C$ and diameter $D$. Let $A(C, D)$ be the minimum area for given $C$ and $D$.

Kubota [6,7] has shown that for $2 D \leqq C \leqq 3 D, \Re(C, D)$ is a 
triangle with sides $D, D$, and $C-2 D$. The corresponding area inequality,

$$
4 A \geqq(C-2 D)\left(4 C D-C^{2}\right)^{1 / 2},
$$

holds even for $C>3 D$. Kubota also establishes for any convex body the inequality

$$
4 A \geqq 3^{1 / 2} D(C-2 D) .
$$

For $C=\pi D, \Re(C, D)$ is a Reuleaux triangle (see Theorem IV 4.1) and

$$
2 A(C, D)=D^{2}\left(\pi-3^{1 / 2}\right) .
$$

Thus the nature of $\Re(C, D)$ remains in doubt only for $3 D<C<\pi D$. In the remainder of the chapter this inequality is assumed to hold.

It is well known $[1, \S 64]$ that each planar set $\Omega$ of diameter $D$ is a subset of an orbiform $\Im$ of width $D$ and that $\Im$ can be chosen so that its interior contains a point of $\Omega$ whose distance from other points of $\Omega$ is less than $D$. The following theorem is a consequence of this result and of Theorem VII 4.1.

TheOREM 1. $\Re(C, D)$ is a cutting body of an orbiform of width $D$. Each extreme point of $\Re(C, D)$ is an end point of a major chord of length $D$.

From Theorem IV 3.1, we have the following theorem.

Theorem 2. $\Re(C, D)$ is a triarc. We may assume $\Re(C, D)$ is a proper triarc.

Since $\Omega(C, D)$ is a triarc, we may make Theorem 1 more explicit. Assume, say, that it is a 3 -arc $L M N$ such that no side of triangle $L M N$ equals $D$. Let $\mathfrak{S}$ be a principal $C C$-support of $\Re(C, D)$. Let $L_{1}$ be the first point of $\AA^{0}(C, D)$ going positively from $M$ such that $L_{1}$ lies on $\mathfrak{S}^{0}$ and $L L_{1}=D$. Let $M_{1}$ and $N_{1}$ be similarly defined. Let the circular arcs of radius $D$ centered at $L$ and $N_{1}$ meet at $L_{2}$. Let $M_{2}$ and $N_{2}$ be similarly defined. We thus obtain $L N_{1} N_{2} M L_{1} L_{2} N M_{1} M_{2} L$, a Reuleaux nonagon containing $\Omega(C, D)$. If one or more sides of $\triangle L M N$ equal $D$, the construction is modified and we obtain the following result. or 9.

TheOREM 3. $\mathfrak{\Re}(C, D)$ is a cutting body of a Reuleaux $n$-gon for $n=3,5,7$,

From the preceding theorem we know that $\Re^{0}(C, D)$ is made up of segments and circular arcs of radius $D$. We continue by showing that the presence of circular arcs is impossible.

Since $C<\pi D, \Omega^{0}(C, D)$ contains at least one segment $P R$. Let $\Gamma_{1}$ be the circular arc of radius $D$ exterior to $\Omega(C, D)$ with end points $P$ and $R$. It is clear from Theorem 3 that the convex hull of $\Re(C, D)$ and $\Gamma_{1}$ has diameter $D$. Let $Q$ be a point on $\Gamma_{1}$. Let arcs $P Q$ and $Q R$ subtend angles of $2 \alpha$ and $2 \beta$ respectively. Let $\alpha+\beta=\gamma$. Triangle $P Q R$ has area $D^{2}(\sin 2 \alpha+\sin 2 \beta-\sin 2 \gamma) / 2$. $P Q+Q R-P R=2 D(\sin \alpha+\sin \beta-\sin \gamma)$. The limit of the ratio of these 
two quantities as $\alpha \rightarrow 0$ and as $\gamma$ remains constant is $D(1+\cos \gamma)$. This is the value of the derivative $d A / d C$ as we start to deform $\Re(C, D)$ by replacing $P R$ by segments $P Q$ and $Q R$.

Assume now that $\Omega^{0}(C, D)$ contains a circular arc $\Gamma_{2}$. If we deform $\Gamma_{2}$ by replacing one of its subarcs $S T$ by the chord $S T$, subtending an angle $2 \theta$, we remove an area of $D^{2}(2 \theta-\sin 2 \theta) / 2$ and shorten the arc by the quantity $2 D(\theta-\sin \theta)$. The limit of the ratio as $\theta \rightarrow 0$ is $2 D$.

If the deformations of the previous paragraphs are carried out simultaneously so that the perimeter remains fixed, the area $A$ decreases initially at the rate of $2 D-D(1+\cos \gamma)$. This is a contradiction and the following theorem therefore holds.

TheOREM 4. $\Re(C, D)$ is a polygon.

It is now natural to conjecture that $\Re(C, D)$ is a triarc $R S T$ in the form of a polygon inscribed in the Reuleaux triangle $R S T$. Assuming the truth of the conjecture, a much more accurate description of $\Omega(C, D)$ can be given. It remains doubtful, however, whether for $3 D<C<\pi D$ a simple inequality giving lower bounds for $A$ in terms of $C$ and $D$ exists which is better than the second Kubota inequality noted at the beginning of the chapter.

\section{REFERENCES}

1. T. Bonnesen and W. Fenchel, Theorie der konvexen Körper, reprinted, New York, 1948.

2. J. Favard, Problèmes d'extremums relatifs aux courbes convexes, I, II, Ann. École Norm. vol. 46 (1929) pp. 345-369; vol. 47 (1930) pp. 313-324. 27-34.

3. S. Fukasawa, Ein Maximum-problem der Eilinien, Tôhoku Math. J. vol. 26 (1926) pp.

4. T. Hayashi, The extremal chords of an oval, Tôhoku Math. J. vol. 22 (1923) pp. 387-393. 50-57.

5. S. Kawai, An inequality for the closed convex curve, Tôhoku Math. J. vol. 36 (1932) pp.

6. T. Kubota, Einige Ungleichheitsbeziehungen über Eilinien und Eiflächen, The Science Reports of the Tôhoku Imperial University Ser. 1 vol. 12 (1923) pp. 45-65.

7. - Eine Ungleichheit für Eilinien, Math. Zeit. vol. 20 (1924) pp. 264-266.

8. H. Lebesgue, Sur le problème des isopérimètres et sur les domaines de largeur constante, Bull. Soc. Math. France C. R. (1914) pp. 72-76.

9. - Sur quelques questiones de minimum, relatives aux courbes orbiformes, et sur leur rapports avec le calcul des variations, J. Math. Pures Appl. (8) vol. 4 (1921) pp. 67-96.

10. M. Yamanouti, Notes on closed convex figures, Proceedings of the Physico-Mathematical Society of Japan (3) vol. 14 (1932) pp. 605-609.

Brown UNIVERSITY, Providence, R. I.

WASHINGTON UNIVERSITY, ST. Louis, Mo. 